This article is forthcoming in

Journal of Experimental Psychology: General

\title{
Science beliefs, political ideology, and cognitive sophistication
}

\author{
Gordon Pennycook ${ }^{1,2 \dagger}$, Bence Bago ${ }^{3}, \&$ Jonathon McPhetres ${ }^{4}$ \\ ${ }^{1}$ Hill/Levene Schools of Business, University of Regina \\ ${ }^{2}$ Department of Psychology, University of Regina \\ ${ }^{3}$ Institute for Advanced Study, Toulouse \\ ${ }^{4}$ Psychology Department, Durham University \\ †Corresponding author: gordon.pennycook@ uregina.ca
}

It is often assumed that a primary source of contention surrounding science is political and, therefore, that partisan disagreement drives attitudes about various science topics. Other models focus on the roles of basic science knowledge and cognitive sophistication, arguing that these facilitate pro-science beliefs. To test these competing accounts, we identified a range of controversial issues ostensibly subject to potential ideological disagreement and examined the relative roles of political ideology, science knowledge, and cognitive sophistication. Results show there was actually very little partisan disagreement on a wide range of nonetheless contentious scientific issues. We also found only weak evidence for identity-protective cognition; instead, reasoning ability was broadly associated with pro-science beliefs. Two experiments that focused specifically on anthropogenic climate change found that increasing political motivations did not increase polarization among individuals who are higher in cognitive sophistication, indicating that increasing political motivations may not have as straightforward of an impact in this context as often assumed. Finally, one's level of basic science knowledge was the most consistent predictor of people's beliefs about science across a wide range of issues. Results suggest that educators and policymakers should focus on increasing basic science literacy and critical thinking rather than the ideologies that purportedly divide people.

Keywords: science beliefs; science attitudes; political ideology; cognitive sophistication; cognitive reflection; motivated reasoning; identity-protective cognition 


\section{Introduction}

Anti-science attitudes represent a major roadblock for responsible public policy. Suitably, a great deal of research has focused on building a better understanding of why people believe what they believe about science. One of the most salient features of this scholarship is the focus on the apparently critical role of politics in science (Bohr, 2014; Ehret et al., 2017; Gauchat, 2012; Joslyn \& Sylvester, 2019; Kahan, 2013; McCright et al., 2016; Mccright \& Dunlap, 2011; Pennycook et al., 2020; Rutjens et al., 2017, 2018; Scheufele, 2013, 2014; van der Linden et al., 2017; Wood \& Porter, 2019). In particular, political ideology is viewed as a central predictor of one's attitudes toward science (Gauchat, 2012; Iyengar \& Massey, 2019; Joslyn \& Sylvester, 2019; Kahan et al., 2017; Scheufele, 2014) and a prominent theory in the context of science beliefs, identity-protective cognition, argues that partisan differences are actually exacerbated by reasoning capacity (Drummond \& Fischhoff, 2017b; Kahan, 2015; Kahan, 2013; Kahan et al., 2012). This rising tide of research has major implications for science communication and education. If politics is so severely clouding science, then focusing on increasing people's basic science knowledge (Allum et al., 2011) and analytic thinking skills (Pennycook, Fugelsang, et al., 2015) is unlikely to diminish anti-science beliefs.

Our goal here is to critically evaluate this zeitgeist in the literature. Most notably, much of the research in this domain focuses on a small handful of scientific issues - namely, beliefs about anthropogenic climate change (e.g., Kahan et al., 2012), evolution (e.g., Drummond \& Fischhoff, 2017a; Rutjens et al., 2017), and vaccinations (e.g., Joslyn \& Sylvester, 2019). These specific research programs are then generalized into much larger conclusions about the role of political ideology (and related mechanisms) in anti-science attitudes writ large. To take an example, a single study on climate change may be distilled into a statement such as 
“...minimizing the salience of partisanship...can offer a useful strategy for improving public understanding of contentious scientific information..." (Guilbeault et al., 2018). Relatedly, the claim that politically motivated reasoning is responsible for perverting scientific conclusions and overwhelms science communication (Iyengar \& Massey, 2019) is based on a few select issues, but is nonetheless may be generalized to science communication more broadly. Even research that claims to test both controversial and non-controversial topics (Allum et al., 2011; Drummond \& Fischhoff, 2017a) often only focuses on a very limited set of science topics. This tendency to focus on a small handful of potentially non-representative issues severely undermines our ability to come to conclusions about the underlying mechanisms that produce anti-science attitudes. The literature may therefore be painting a distorted picture of the correlates of people's beliefs about science. This problem is compounded by a lack of studies that have simultaneously tested multiple competing accounts. Therefore, based on the literature at present, it is hard to know which factors account for the most relative variance in anti-science beliefs across various science-related issues.

To address these issues, it is necessary to expand the investigation of science-beliefs to a wider array of topics. In the present work, we simultaneously test multiple accounts of science beliefs using the same methodology across multiple controversial scientific issues. This allows us to not only make broader generalizations about the underlying factors that relate to antiscience beliefs - which is important if we are to develop informed public policy on science communication - but also to test the relative role of different factors, such as political ideology versus basic science knowledge. Instead of looking for evidence that a particular account of science-beliefs is correct, here we focus on which of four different accounts is able to explain the 
most variability in the widest array of controversial science-beliefs. This approach, we hope, is able to mutually inform theory and policy.

Four possible explanations of science attitudes. Several competing (and non-competing) mechanisms have been proposed to explain why people believe what they believe about science. One prominent account - which we will refer to here as the politically motivated reasoning account - argues that ideology biases information processing and therefore plays a causal role in the formation of beliefs (Kunda, 1990; Taber et al., 2009; Taber \& Lodge, 2012). This account is commonly evoked to explain partisan differences in beliefs (or skepticism) about anthropogenic global warming, with conservatism typically being associated with an anti-science stance (Bohr, 2014; Gauchat, 2012; Lewandowsky et al., 2013). This account surfaces the importance of the cultural context of our beliefs. For example, global warming was politicized by conservative think tanks and political elites (Mccright \& Dunlap, 2011) which was then picked up by laypeople who are motivated to believe information that is consistent with their political ideology (Kahan et al., 2012). The implication of a focus on politically motivated reasoning in the context of science beliefs is that simply knowing one's political ideology ought to go a long way in predicting what they believe about science. ${ }^{1}$

This general focus on the importance of political motivations is also a prominent feature of the identity-protective cognition account (Kahan, 2013; Kahan et al., 2012). However, in contrast to broader accounts of politically motivated reasoning - which describe motivated reasoning as

\footnotetext{
${ }^{1}$ Note that other ideological factors, such as religiosity, also be implicated in motivated reasoning processes. For example, religious people may be motivated to reject evolution based on theological teachings (Hill, 2014). Nonetheless, we focus here on politically motivated reasoning in the context of science beliefs and thus our data do not speak directly to other potential motivational effects or to the importance of motivated reasoning for other (non-science) beliefs more broadly (see Discussion for further on this issue).
} 
being driven by largely automatic and affect process (Erisen et al., 2014; Lodge \& Taber, 2005)

- identity-protective cognition contends that cognitively sophisticated individuals are actually better able to use their reasoning skills to selectively conform their evaluation of evidence to their political ideology (Kahan, 2013; Kahan et al., 2012). Indeed, research has claimed that polarization around scientific issues is stronger (not weaker) among individuals who are more cognitively sophisticated and/or educated (Drummond \& Fischhoff, 2017a). For example, concern for climate change decreases with increased numeracy among Republicans but the opposite association is evident for Democrats (Kahan et al., 2012), and those with greater science literacy are more polarized on beliefs about stem cell research, the big bang, and evolution (Drummond \& Fischhoff, 2017a). This research implies that politically motivated reasoning is an active reasoning process that is facilitated by deliberative processes, hence why cognitive sophistication is thought to exacerbate political polarization.

However, researchers testing the identity-protective cognition account have focused on a select number of particularly prominent issues (Drummond \& Fischhoff, 2017a; Gauchat, 2012; Kahan et al., 2012). These findings have then been generalized to a wider range of issues. For example, the statement "some of the most polarizing topics in American politics are scientific ones" (Scheufele, 2014) invokes scientific topics more broadly based on the results of studies looking at a few very specific (and potentially outlying) issues, such as climate change, biotechnology, and gun control (Drummond \& Fischhoff, 2017b; Kahan, 2013; Kahan et al., 2012). Thus, it remains unclear if these few issues are the exception or the rule and therefore it is unknown if the identity-protective account can be extended to a wider variety of politicallyrelevant anti-science beliefs. In addition to representing a more rigorous test of the underlying theory, understanding this broader pattern of results is important because it would be problematic 
for broad public policy on science education to be influenced by a few highly salient but exceptional cases.

In contrast to accounts that focus on political motivations and identity, the knowledge deficit account suggests that people reject (or fail to accept) certain scientific claims simply because they do not possess enough (or the correct) basic scientific knowledge (Allum et al., 2011; Lombrozo et al., 2008; Weisberg et al., 2021). Specifically, science knowledge is often considered in terms of the basic facts that one knows (e.g., that electrons are smaller than atoms, or that antibiotics don't kill viruses; see Weisberg et al., 2018) - or in terms of knowledge about the scientific method itself (e.g., knowing the difference between a theory and a hypothesis; see Weisberg et al., 2021). The knowledge deficit model implies that anti-science beliefs are prevalent primarily because science is difficult to understand without advanced training. Indeed, people who (for example) have greater knowledge of how science works have more proscientific beliefs about evolution, climate change, and vaccines (Weisberg et al., 2021). A key policy prescription from this model is that teaching people about science will straightforwardly lead to an increase in pro-scientific beliefs and attitudes.

Closely related is what we will call the analytic thinking account, which is that people simply do not think analytically enough about science (or other) issues (Pennycook, Fugelsang, et al., 2015). Whereas the knowledge deficit account suggests that having a strong core understanding of basic scientific facts is central to the formation of pro-science attitudes, the analytic thinking account argues that the disposition to think analytically and critically (over and above underlying science knowledge or trust in science; O’Brien et al., 2021) is critical. For example, studies have found that those who reason more analytically are more likely to endorse evolution (Gervais, 2015). In contrast, individuals who are more receptive to pseudo-profound 
bullshit—randomly generated nonsense statements—are both less analytic and more likely to believe in the efficacy of non-evidenced based alternative medicines (Pennycook, Cheyne, et al., 2015). As above, however, it is unclear if these results are specific to particular science-related beliefs. Given the cluttered theoretical space surrounding science beliefs, we set out to simultaneously test all four accounts.

Present work. We approached this issue in two ways. First, we set out to examine beliefs about a wide range of scientific topics using a common methodology. We identified a large range of controversial issues on which people believe there is ideological disagreement (see Figure 1). A preregistered pilot test using an independent sample demonstrated that people believe each selected science topic to be politically divisive (albeit to varying degrees; see Tables S1-S2). Of course, this does not mean that the topics actually are divisive, nor that they are subject to politically motivated reasoning effects. We then introduced an experimental manipulation of political motivations on a targeted issue that showed the strongest evidence of political differences (namely, climate change) to elucidate potential mechanisms.

To test each theoretical possibility across the full range of scientific issues, we derived specific predictions from the four accounts of science attitudes described above. The politically motivated reasoning account predicts that ideology will be a strong and consistent correlate of science attitudes and the knowledge deficit account makes the same prediction, but for basic science knowledge instead of ideology. Although these accounts are not all mutually exclusive, our goal is to assess which explains more variance and for which science topics. The identityprotective cognition and analytic thinking accounts do, however, make competing predictions: Whereas the former predicts that cognitive sophistication will be associated with increased political polarization, the latter predicts that cognitive sophistication will simply be associated 
with increased pro-scientific attitudes across the board (for a similar approach see Taber et al., 2009). Although it is possible that each of the four accounts is the best explanation for single specific science-related beliefs, it is crucial to ascertain which account(s) hold the strongest (and broadest) predictive power. This will help guide informed public policy in addition to a greater understanding of human cognition. We tested these predictions using two samples of Americans.

The data from our two samples in Study 1 found strong support for the knowledge deficit and analytic thinking accounts and surprisingly little support for the motivated reasoning account and identity-protective cognition accounts. To delve deeper into this, we conducted two experiments where we asked participants directly to evaluate arguments about climate change (a particularly divisive issue) in a partisan way. Critically, we found that cognitive sophistication was, if anything, associated with decreased polarization under partisan motivation instructions. Thus, we find a consistent pattern across several scientific issues (in Study 1) and in experimental approaches to a specific scientific issue (in Studies 2 and 3).

Our analysis plans, inclusion criteria, and study design were preregistered prior to data collection; all data and materials are available on the Open Science Framework (https://osf.io/h4ej5/?view_only=a96965ef6e8342eab99fe446d3fba198). The preregistration for Sample 1 is available at: https://osf.io/s9n24/?view_only=06204d2bc9ea496db7d80fa15706aa2b. The preregistration for Sample 2 is available at: https://osf.io/5tr2f/?view_only=ea980de25a68437c928211b2b1d01a6d. The preregistration for the pilot sample is available at: https://osf.io/wsz7e/?view_only=d13810b9e9ca40a9adbc269a019e97f8. The preregistrations for Studies 2 and 3 are available at: https://osf.io/h4ej5/?view_only=a96965ef6e8342eab99fe446d3fba198. 


\section{Study 1}

We begin by investigating the variance explained by the four accounts of science beliefs across a wide variety of ostensibly politically divisive issues.

\section{Method}

We conducted this survey twice using two sample sources that closely match the demographics of the United States. For Sample 1, we recruited subjects through Lucid, an online panel company which provides samples that closely match U.S. demographics on age, gender, region, and ethnicity using quota-sampling. We then conducted an identical survey using an independent sample to examine the replicability of our results. For Sample 2, we recruited subjects through Dynata, an online panel company which provides representative samples that are quota-matched for United States demographics based on age, gender, education, income, and political party affiliation. The survey procedures for both studies were identical and the results are described in tandem, below.

\section{Participants}

Sample 1. We recruited 1,003 subjects from Lucid. Complete demographics are presented in Table S3. Following our preregistered plans, we excluded 312 subjects for failing at least one of two attention checks, resulting in 691 subjects in the final dataset. A sensitivity analyses indicates this is sufficient to detect effects of $r=.13$ or larger with .95 power.

Sample 2. We recruited 1,018 subjects from Dynata. Complete demographics are presented in Table S3. While we preregistered the same exclusion criteria as with Sample 1, nearly $50 \%$ of the sample ( $n=453)$ failed at least one of these attention checks. Thus, in order to present the most conservative results, we deviated from our plan and did not implement any exclusion criteria (we report results of key analyses using preregistered exclusion criteria in 
Supplementary Materials file 2). However, zero-order correlations and scale reliabilities were extremely similar between those who met and did not meet the inclusion criteria.

\section{Materials and Procedure}

Subjects completed an online survey hosted on the Qualtrics platform. The order of all survey scales was randomized with the constraint that questions about science beliefs were always first. The survey for Sample 1 also included questions about natural and organic foods and the survey for Sample 2 included questions about religiosity and biblical literacy for the purposes of other studies; these questions always came last. At the end of the survey, subjects completed demographics questions. Descriptive statistics and reliabilities for each scale are displayed in Table 1.

Pro-science beliefs. Subjects answered questions about their agreement with 17 statements about various scientific and pseudoscientific topics on a scale of 1 (strongly disagree) to 6 (strongly agree). A preregistered pilot sample demonstrated that all topics were believed to be divisive (see Tables S1-S2).

We also created two subsets of these items based on how they were generated; liberal proscience beliefs included belief in global warming, evolution, the big bang, and sex ambiguity. Conservative pro-science items comprised the remainder of the items (see Figure 1). Naturally, both sets of items could fit into either category depending on the valence of the item wording our labels are intended to identify the issues for which liberals or conservatives are most likely to hold the pro-scientific stance based on the cultural narratives that were evident when the items were created. Put differently, the scales are determined based on the apparent source of politicization. In addition, we over-sampled ostensibly conservative pro-science issues in an 
attempt to counterbalance the established (and strong) ideological differences on global warming, evolution, and big bang (liberal pro-science items).

Science knowledge. Subjects answered 12 questions about basic (and general) science knowledge (e.g., "Antibiotics can cure viruses as well as bacteria"; "The centre of the earth is very hot") taken from previous surveys, correct answers were summed. We excluded any items that were related to the pro-science belief questions (e.g., questions about global warming, evolution, genetics, etc).

Science methodology. There were also 9 multiple choice questions assessing knowledge about scientific methodology (e.g., "What is the difference between a theory and a hypothesis?").

Cognitive sophistication. We selected 3 measures to capture different dimensions of cognitive sophistication. These measures have been used in a variety of recent research and approximate similar aspects of this construct (Pennycook et al., 2020; Tappin et al., 2020b). First, we used a 6-item Cognitive Reflection Test (CRT; (Frederick, 2005). The CRT poses problems with an incorrect intuitive answer and is intended to measure the disposition to engage in analytic thinking (Pennycook, Fugelsang, et al., 2015). Our CRT was a 6-item scale because the 'hole' item, which did not contribute to scale reliability in our previous work, was not included. Questions were presented in open-ended format; correct responses were coded as 1 and the 6 items were averaged together; higher scores represent greater analytic thinking.

The 8-item actively open-minded thinking about evidence (AOT-E) scale (Pennycook et al., 2020), which measures the degree to which one is open to information contradicting their opinions (e.g., "People should always take into account evidence that goes against their opinions"). Items were rated on a scale from 1 (strongly disagree) to 6 (strongly agree); higher scores represent a stronger belief that their beliefs should change according to evidence. 
The bullshit receptivity scale (BSR; Pennycook, Cheyne, et al., 2015) measures the extent to which people find randomly generated (bullshit) statements as representing something profound. Participants rated the profoundness of five statements (e.g. "Imagination is inside exponential space time events") on a scale from 1 (not at all profound) to 5 (very profound); higher scores represent greater receptivity to bullshit.

\section{Results}

Which issues are politically partisan? The items and distributions according to political partisanship are displayed in Figure 1. As can be seen, the distribution of agreement ratings was remarkably similar between Republicans and Democrats, suggesting that there is little partisan disagreement (with the exception of a few select issues). This occurred despite the fact that we explicitly sampled issues that the majority of an out-of-sample group rated as being subject to political disagreement (see Table S2). Furthermore, as is evident from Figure 1, most of the issues were highly contentious. That is, there was a wide spread in opinions about the issues and anti-science beliefs were common. 
Figure 1. Science belief items and distribution according to political partisanship for Samples 1 and 2. The left half depicts distributions for Sample $1(N=691)$, and the right half depicts distributions for Sample $2(N=1,018)$; *indicates the item was reversed for subsequent analyses.
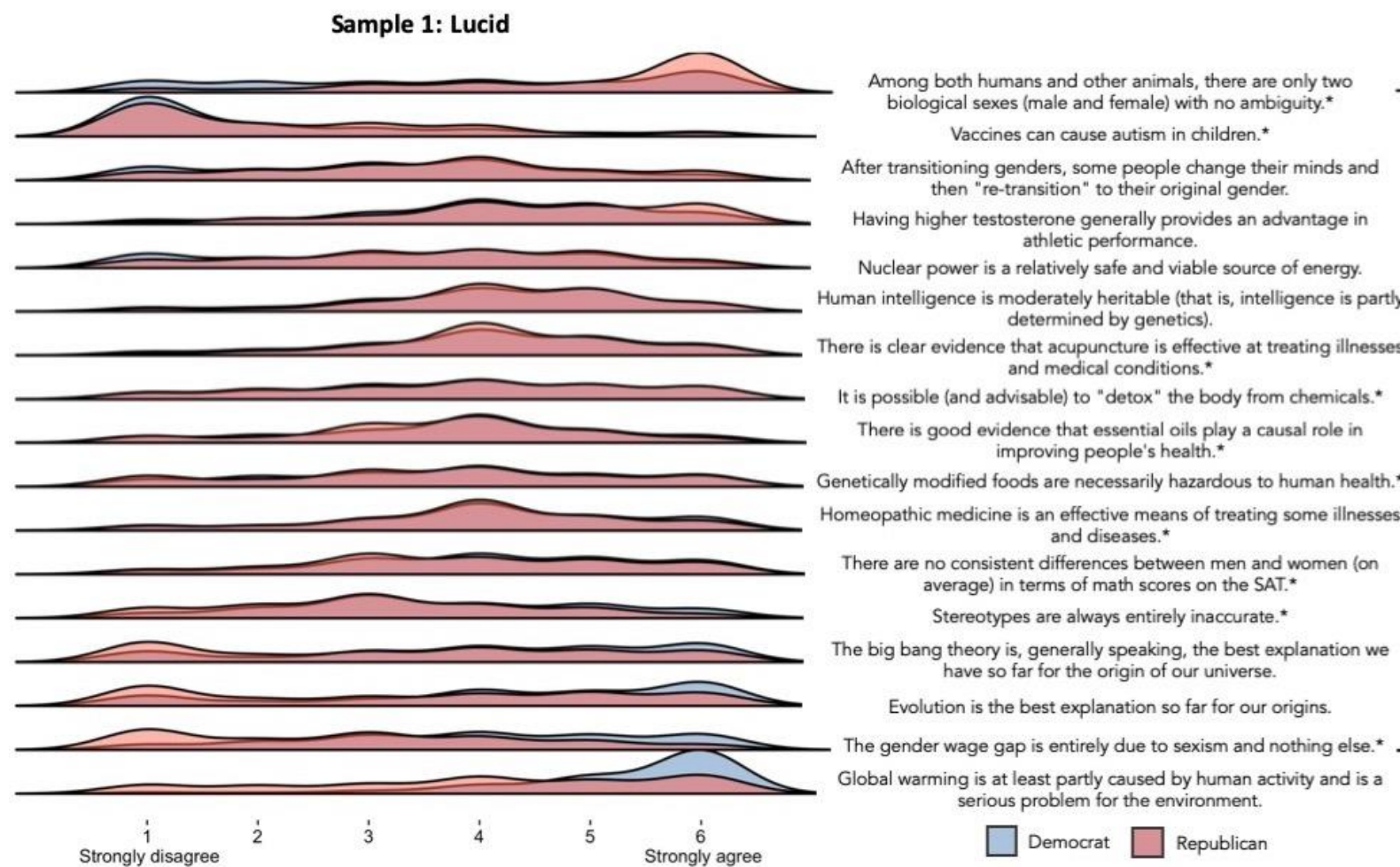

Among both humans and other animals, there are only two biological sexes (male and female) with no ambiguity.* Vaccines can cause autism in children. ${ }^{*}$ After transitioning genders, some people change their minds and then "re-transition" to their original gender. Having higher testosterone generally provides an advantage in (n) viable source of energy. Human intelligence is moderately heritable (that is, intelligence is partly

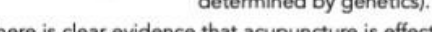
There is clear evidence that acupuncture is effective at treating illnesses

It is possible (and advisable) to "detox" the body from chemicals. There is good evidence that essential oils play a causal role in
improving people's health.* Genetically modified foods are necessarily hazardous to human health. Homeopathic medicine is an effective means of treating some illnesses There are no consistent differences between men and women (on average) in terms of math scores on the SAT." Stereotypes are always entirely inaccurate. * The big bang theory is, generally speaking, the best explanation we have so far for the origin of our universe.

Evolution is the best explanation so far for our origins. The gender wage gap is entirely due to sexism and nothing else.* Global warming is at least partly caused by human activity and is a srous problem for the environment.

$\square$ Democrat $\square$ Republican

Strongly disagree

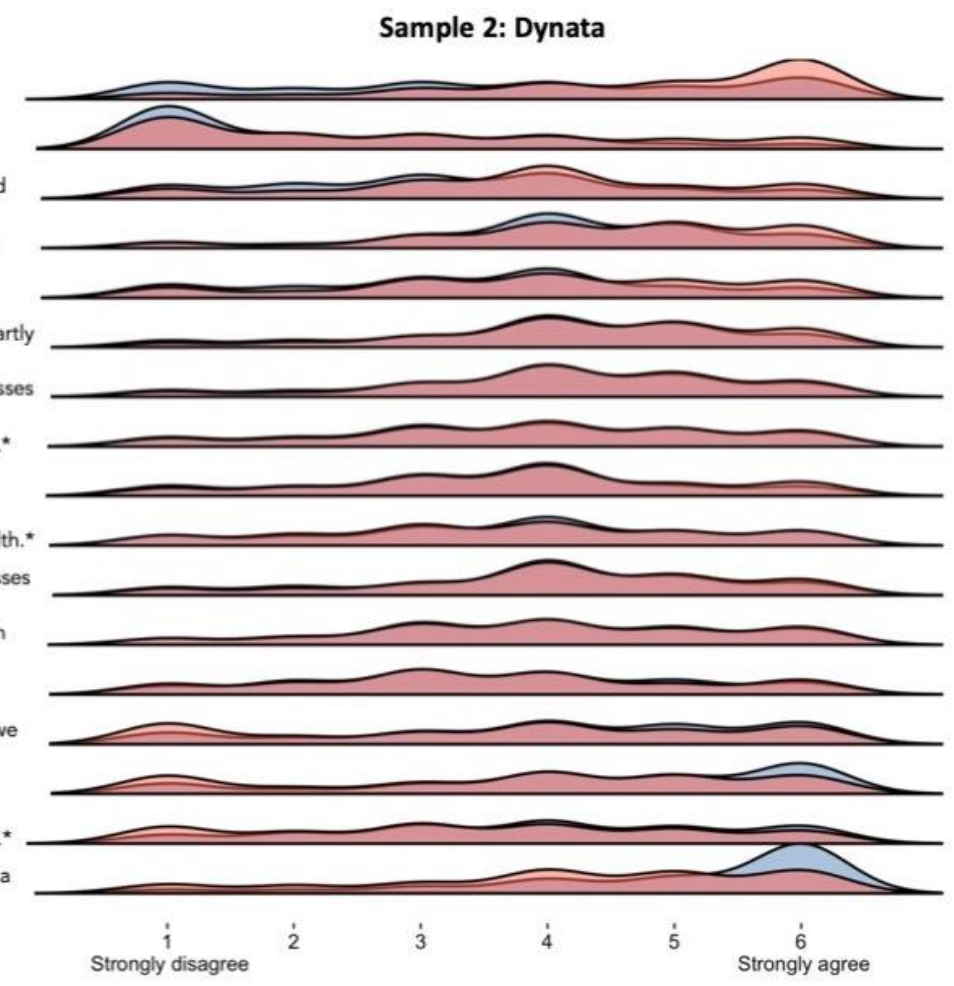


For all subsequent analyses, items were recoded such that a higher score accords with a more 'pro-science' position. We also created two political subsets of these items based on how they were generated; ostensibly liberal pro-science beliefs included belief in global warming, evolution, the big bang, and sex ambiguity. Ostensibly conservative pro-science items comprised the remainder of the items (we over-sampled ostensibly conservative pro-science issues in an attempt to counterbalance the established - and strong - ideological differences on global warming, evolution, and big bang). We then examined zero-order correlations between all measures (see Table 1).

The pattern of results from the aggregate measures of science belief broadly support the knowledge deficit and analytic thinking accounts. First, there is some heterogeneity across science topics (as indicated by the low reliability values), suggesting that there are a variety of reasons why people hold the beliefs they do (as opposed to a dominant ideological reason). Second, the pro-science beliefs composite was positively correlated with science knowledge ( $r$ 's $=.36, .37)$, knowledge of scientific methodology $(r ' s=.27, .31)$, actively open-minded thinking $(r ' s=.33, .40)$, and higher scores on the CRT (i.e., more reflective thinking) $(r$ 's $=.36, .34)$. These effect sizes are relatively large and, in fact, range from the $70^{\text {th }}$ to $90^{\text {th }}$ percentile based on established norms in social psychology (Funder \& Ozer, 2019; Gignac \& Szodorai, 2016). Proscience beliefs were also negatively correlated with religiosity $(r$ 's $=-.44)$, political ideology/partisanship (indicating lower overall acceptance of science among political conservatives) ( $r$ 's $=-.14,-.15)$, and bullshit receptivity ( $r$ 's $=-18,-.34)$. Interestingly, CRT performance and BSR were correlated with conservative but not liberal pro-science beliefs, again indicating heterogeneity across issues. 
Table 1. Reliabilities, means, standard deviations, and correlations. Top half refers to Sample 1 (Lucid; $N=691$ ) and bottom half refers to Sample 2 (Dynata; $N=1,018$ ); $M$ and $S D$ refer to mean and standard deviation, respectively; $\alpha$ indicates Cronbach's alpha; for political ideology and partisanship, a lower score indicates liberal/Democrat and a high score indicates conservative/Republican; AOT-E $=$ actively open-minded thinking about evidence; $\mathrm{CRT}=$ cognitive reflection test; $\mathrm{BSR}=$ bullshit receptivity; $* p<.05$. $* * p<$ .01 .

\begin{tabular}{|c|c|c|c|c|c|c|c|c|c|c|c|c|c|c|c|c|c|}
\hline \multirow[b]{2}{*}{ Variable } & \multicolumn{3}{|c|}{ Sample 1: Lucid } & \multicolumn{3}{|c|}{ Sample 2: Dynata } & \multirow[b]{2}{*}{1} & \multirow[b]{2}{*}{2} & \multirow[b]{2}{*}{3} & \multirow[b]{2}{*}{4} & \multirow[b]{2}{*}{5} & \multirow[b]{2}{*}{6} & \multirow[b]{2}{*}{7} & \multirow[b]{2}{*}{8} & \multirow[b]{2}{*}{9} & \multirow[b]{2}{*}{10} & \multirow[b]{2}{*}{11} \\
\hline & $\alpha$ & M & $\mathrm{SD}$ & $\alpha$ & M & $\mathrm{SD}$ & & & & & & & & & & & \\
\hline 1. Pro-science beliefs (total) & 0.51 & 3.57 & 0.51 & 0.43 & 3.58 & 0.48 & & $.54 * *$ & $.83 * *$ & $.36^{* *}$ & $.27 * *$ & $-.44 * *$ & $-.14 * *$ & $-.11 * *$ & $.33 * *$ & $.36 * *$ & $-.18 * *$ \\
\hline 2. Liberal pro-science beliefs & 0.63 & 3.73 & 1.2 & 0.60 & 3.84 & 1.12 & $.41 * *$ & & -0.01 & $.18 * *$ & $.10^{* *}$ & $-.44 * *$ & $-.46^{* *}$ & $-.46 * *$ & $.27 * *$ & 0.06 & 0.07 \\
\hline 3. Conserv. pro-science beliefs & 0.53 & 3.52 & 0.56 & 0.54 & 3.5 & 0.58 & $.84 * *$ & $-.15^{* *}$ & & $.31 * *$ & $.26 * *$ & $-.23 * *$ & $.13 * *$ & $.18 * *$ & $.22 * *$ & $.39 * *$ & $-.26^{* *}$ \\
\hline 4. Science knowledge & 0.68 & 7.29 & 2.59 & 0.76 & 6.62 & 3.04 & $.37 * *$ & $.20 * *$ & $.28 * *$ & & $.46 * *$ & $-.14 * *$ & -0.03 & -0.02 & $.39 * *$ & $.43 * *$ & $-.18 * *$ \\
\hline 5. Science methodology & 0.64 & 5.87 & 1.96 & 0.7 & 5.27 & 2.33 & $.31 * *$ & $.10 * *$ & $.28 * *$ & $.51 * *$ & & $-.09 *$ & -0.06 & -0.06 & $.42 * *$ & $.37 * *$ & $-.27 * *$ \\
\hline 6. Religiosity & 0.95 & 5.11 & 1.67 & 0.95 & 4.94 & 1.71 & $-.44 * *$ & $-.35 * *$ & $-.27 * *$ & $-.15^{* *}$ & $-.12 * *$ & & $.28 * *$ & $.21 * *$ & $-.25 * *$ & $-.22 * *$ & $.17 * *$ \\
\hline 7. Political ideology & 0.89 & 4.12 & 1.58 & 0.87 & 4.13 & 1.53 & $-.15 * *$ & $-.32 * *$ & 0.03 & 0.02 & -0.05 & $.34 * *$ & & $.67 * *$ & $-.20 * *$ & 0.02 & -0.02 \\
\hline 8. Political partisanship & - & 3.39 & 1.56 & - & 3.34 & 1.52 & $-.14 * *$ & $-.32 * *$ & 0.04 & 0 & -0.06 & $.29 * *$ & $.60 * *$ & & $-.15^{* *}$ & 0.06 & -0.05 \\
\hline 9. AOT-E & 0.74 & 4.33 & 0.83 & 0.72 & 4.2 & 0.84 & $.40 * *$ & $.31 * *$ & $.25 * *$ & $.49 * *$ & $.53^{* *}$ & $-.24 * *$ & $-.13 * *$ & $-.17 * *$ & & $.33 * *$ & $-.23 * *$ \\
\hline 10. CRT & 0.72 & 0.35 & 0.29 & 0.73 & 0.3 & 0.28 & $.34 * *$ & $.13^{* *}$ & $.30 * *$ & $.47 * *$ & $.48 * *$ & $-.18 * *$ & -0.05 & -0.01 & $.41 * *$ & & $-.26 * *$ \\
\hline 11. BSR & 0.82 & 2.83 & 0.96 & 0.88 & 2.89 & 1.06 & $-.34 * *$ & $.11^{* *}$ & $-.44 * *$ & $-.17 * *$ & $-.34 * *$ & $.23 * *$ & 0.04 & 0.03 & $-.26 * *$ & $-.31 * *$ & \\
\hline
\end{tabular}


Next, we examined correlations between our predicting variables and each science topic individually (see Table 2). These results plainly show that each measure was broadly predictive for a number of issues (to varying degrees), but that there was a great deal of variation between science issues. This demonstrates how the past literature on science attitudes - which has focused almost exclusively on a few key issues - has failed to represent the apparently multifaceted nature of science acceptance or rejection. For example, although the issues are ostensibly political, ideology and partisanship had moderate-to-strong correlations $(r$ 's $>.20$; see (Funder \& Ozer, 2019) with only two items across both samples consistently (global warming and binary sex ambiguity); the other 15 items were more weakly correlated or even uncorrelated with ideology. Put differently, correlations were moderate-to-strong in 8 only out of 34 possible cases for ideology or partisanship. Nonetheless, it was the case the political ideology and partisanship significantly correlated with beliefs (to some extent) across most issues; however, as is also evident from Table 2, this was the case for our measures of science knowledge and cognitive sophistication as well. 
Table 2. Zero-order correlations (Pearson's $r$ ) for each science topic for both samples. Lucid $(N=691)$; Dynata $(N=1,018)$;

Higher scores are more pro-science for each topic; for political ideology/partisanship, a lower score indicates liberal/Democrat and a high score indicates conservative/Republican; AOT-E = actively open-minded thinking about evidence; CRT $=$ cognitive reflection test; BSR = bullshit receptivity; values in square brackets indicate the $95 \%$ confidence interval for each correlation. * indicates $p<$ .05 . ** indicates $p<.01$.

\begin{tabular}{|c|c|c|c|c|c|c|c|c|c|c|}
\hline \multirow[t]{2}{*}{ Variable } & \multicolumn{2}{|c|}{ Global warming } & \multicolumn{2}{|c|}{ Wage gap sexism } & \multicolumn{2}{|c|}{ Sex ambiguity } & \multicolumn{2}{|c|}{ Evolution accuracy } & \multicolumn{2}{|c|}{ Big Bang belief } \\
\hline & Lucid & Dynata & Lucid & Dynata & Lucid & Dynata & Lucid & Dynata & Lucid & Dynata \\
\hline \multirow[t]{2}{*}{ Political Partisanship } & $-.45 * *$ & $-.27 * *$ & $.34 * *$ & $.13^{* *}$ & $-.33 * *$ & $-.31 * *$ & $-.28 * *$ & $-.16 * *$ & $-.25 * *$ & $-.12 * *$ \\
\hline & {$[-.51,-.39]$} & {$[-.33,-.21]$} & {$[.28, .41]$} & {$[.07, .19]$} & {$[-.39,-.26]$} & {$[-.36,-.25]$} & {$[-.35,-.21]$} & {$[-.22,-.10]$} & {$[-.31,-.17]$} & {$[-.18,-.06]$} \\
\hline \multirow[t]{2}{*}{ Political ideology } & $-.41 * *$ & $-.25 * *$ & $.32^{* * *}$ & $.14 * *$ & $-.37 * *$ & $-.35 * *$ & $-.29 * *$ & $-.14 * *$ & $-.22 * *$ & $-.11 * *$ \\
\hline & {$[-.47,-.34]$} & {$[-.31,-.19]$} & {$[.25, .38]$} & {$[.07, .20]$} & {$[-.43,-.30]$} & {$[-.40,-.29]$} & {$[-.36,-.22]$} & {$[-.20,-.08]$} & {$[-.29,-.15]$} & {$[-.17,-.05]$} \\
\hline \multirow[t]{2}{*}{ Science knowledge } & .05 & $.15^{* *}$ & .04 & .04 & $.14^{* *}$ & $.09 * *$ & $.11 * *$ & $.16^{* * *}$ & $.17 * *$ & $.14 * *$ \\
\hline & {$[-.02, .13]$} & {$[.09, .21]$} & {$[-.03, .11]$} & {$[-.03, .10]$} & {$[.07, .22]$} & {$[.03, .15]$} & {$[.04, .19]$} & {$[.10, .22]$} & {$[.10, .25]$} & {$[.08, .20]$} \\
\hline \multirow[t]{2}{*}{ Science methodology } & $.11^{* *}$ & $.17 * *$ & .07 & $.11 * *$ & $.13 * *$ & $.10^{* *}$ & .04 & .05 & .01 & -.04 \\
\hline & {$[.04, .19]$} & {$[.11, .23]$} & {$[-.00, .14]$} & {$[.05, .17]$} & {$[.06, .20]$} & {$[.04, .16]$} & {$[-.04, .11]$} & {$[-.02, .11]$} & {$[-.07, .08]$} & {$[-.10, .03]$} \\
\hline \multirow[t]{2}{*}{ Religiosity } & $-.13 * *$ & -.01 & $.07 *$ & $-.07 *$ & $-.21 * *$ & $-.38 * *$ & $-.45^{* *}$ & $-.29 * *$ & $-.41 * *$ & $-.23 * *$ \\
\hline & {$[-.20,-.05]$} & {$[-.08, .05]$} & {$[.00, .15]$} & {$[-.13,-.01]$} & {$[-.28,-.14]$} & {$[-.43,-.32]$} & {$[-.51,-.39]$} & {$[-.35,-.23]$} & {$[-.47,-.35]$} & {$[-.29,-.17]$} \\
\hline \multirow[t]{2}{*}{ AOT-E } & $.25^{* *}$ & $.28 * *$ & -.02 & .01 & $.21 * *$ & $.21 * *$ & $.15^{* *}$ & $.20 * *$ & $.14 * *$ & $.14 * *$ \\
\hline & {$[.18, .32]$} & {$[.23, .34]$} & {$[-.09, .06]$} & {$[-.05, .07]$} & {$[.14, .28]$} & {$[.15, .27]$} & {$[.08, .22]$} & {$[.14, .25]$} & {$[.07, .22]$} & {$[.08, .20]$} \\
\hline \multirow[t]{2}{*}{ CRT } & -.01 & $.09 * *$ & $.12 * *$ & $.09^{* *}$ & $.09 *$ & $.14 * *$ & .02 & $.06^{*}$ & .07 & $.07 *$ \\
\hline & {$[-.08, .06]$} & {$[.02, .15]$} & {$[.04, .19]$} & {$[.02, .15]$} & {$[.01, .16]$} & {$[.08, .20]$} & {$[-.05, .10]$} & {$[.00, .12]$} & {$[-.00, .15]$} & {$[.00, .13]$} \\
\hline \multirow[t]{2}{*}{ BSR } & $.11^{* *}$ & $.13 * *$ & $-.14 * *$ & $-.27 * *$ & -.05 & $-.17 * *$ & $.08^{*}$ & $.15^{* *}$ & .05 & $.20 * *$ \\
\hline & {$[.03, .18]$} & {$[.07, .19]$} & {$[-.21,-.06]$} & {$[-.33,-.21]$} & {$[-.12, .03]$} & {$[-.23,-.11]$} & {$[.01, .16]$} & {$[.09, .21]$} & {$[-.02, .13]$} & {$[.14, .26]$} \\
\hline
\end{tabular}




\begin{tabular}{|c|c|c|c|c|c|c|c|c|}
\hline \multirow[t]{2}{*}{ Variable } & \multicolumn{2}{|c|}{ Stereotype accuracy } & \multicolumn{2}{|c|}{ Vaccine safety } & \multicolumn{2}{|c|}{ Re-transition gender } & \multicolumn{2}{|c|}{ Gender SAT } \\
\hline & Lucid & Dynata & Lucid & Dynata & Lucid & Dynata & Lucid & Dynata \\
\hline \multirow[t]{2}{*}{ Political Partisanship } & $.14^{* *}$ & .01 & $-.12 * *$ & $-.18^{* *}$ & $.10 * *$ & $.20 * *$ & $.10 *$ & -.01 \\
\hline & {$[.06, .21]$} & {$[-.05, .07]$} & {$[-.19,-.04]$} & {$[-.24,-.12]$} & {$[.03, .18]$} & {$[.14, .26]$} & {$[.02, .17]$} & {$[-.08, .05]$} \\
\hline \multirow[t]{2}{*}{ Political ideology } & $.11^{* *}$ & -.02 & $-.13 * *$ & $-.17 * *$ & $.10^{*}$ & $.22 * *$ & .03 & $<.01$ \\
\hline & {$[.04, .18]$} & {$[-.08, .05]$} & {$[-.20,-.05]$} & {$[-.23,-.11]$} & {$[.02, .17]$} & {$[.16, .28]$} & {$[-.04, .11]$} & {$[-.06, .07]$} \\
\hline \multirow[t]{2}{*}{ Science knowledge } & $.16^{* *}$ & $.14 * *$ & $.21 * *$ & $.25 * *$ & .01 & $<.01$ & $<.01$ & $<.01$ \\
\hline & {$[.09, .24]$} & {$[.08, .20]$} & {$[.14, .28]$} & {$[.19, .31]$} & {$[-.06, .09]$} & {$[-.06, .06]$} & {$[-.07, .08]$} & {$[-.06, .07]$} \\
\hline \multirow[t]{2}{*}{ Science methodology } & $.20 * *$ & $.20 * *$ & $.29 * *$ & $.39 * *$ & -.05 & $-.15^{* *}$ & $<.01$ & .04 \\
\hline & {$[.13, .27]$} & {$[.14, .26]$} & {$[.22, .36]$} & {$[.34, .44]$} & {$[-.12, .03]$} & {$[-.21,-.09]$} & {$[-.08, .07]$} & {$[-.02, .10]$} \\
\hline \multirow[t]{2}{*}{ Religiosity } & -.06 & $-.14 * *$ & $-.19 * *$ & $-.23 * *$ & .06 & $.22 * *$ & -.01 & $-.09 * *$ \\
\hline & {$[-.13, .02]$} & {$[-.20,-.08]$} & {$[-.26,-.11]$} & {$[-.29,-.17]$} & {$[-.01, .14]$} & {$[.16, .28]$} & {$[-.08, .06]$} & {$[-.15,-.03]$} \\
\hline \multirow[t]{2}{*}{ AOT-E } & $.13 * *$ & $.18^{* *}$ & $.24 * *$ & $.39^{* *}$ & -.07 & $-.15 * *$ & -.02 & $<.01$ \\
\hline & {$[.06, .21]$} & {$[.12, .24]$} & {$[.17, .31]$} & {$[.34, .44]$} & {$[-.14, .01]$} & {$[-.21,-.09]$} & {$[-.10, .05]$} & {$[-.06, .07]$} \\
\hline \multirow[t]{2}{*}{ CRT } & $.20 * *$ & $.20 * *$ & $.22 * *$ & $.28 * *$ & -.01 & $-.09 * *$ & .05 & .04 \\
\hline & {$[.13, .27]$} & {$[.14, .25]$} & {$[.15, .29]$} & {$[.23, .34]$} & {$[-.08, .07]$} & {$[-.15,-.03]$} & {$[-.02, .13]$} & {$[-.02, .10]$} \\
\hline \multirow[t]{2}{*}{ BSR } & $-.14 * *$ & $-.31 * *$ & $-.16^{* *}$ & $-.38 * *$ & .05 & $.25^{* *}$ & -.04 & $-.20 * *$ \\
\hline & {$[-.22,-.07]$} & {$[-.36,-.25]$} & {$[-.24,-.09]$} & {$[-.43,-.33]$} & {$[-.02, .13]$} & {$[.19, .30]$} & {$[-.12, .03]$} & {$[-.26,-.14]$} \\
\hline
\end{tabular}




\begin{tabular}{|c|c|c|c|c|c|c|c|c|}
\hline \multirow[t]{2}{*}{ Variable } & \multicolumn{2}{|c|}{ Nuclear Power } & \multicolumn{2}{|c|}{ Testosterone athleticism } & \multicolumn{2}{|c|}{ IQ heritability } & \multicolumn{2}{|c|}{ Homeopathy rejection } \\
\hline & Lucid & Dynata & Lucid & Dynata & Lucid & Dynata & Lucid & Dynata \\
\hline \multirow[t]{2}{*}{$\begin{array}{l}\text { Political } \\
\text { Partisanship }\end{array}$} & $.09 *$ & $.16^{* *}$ & $.09 *$ & $.11 * *$ & $.08 *$ & $.10^{* *}$ & .04 & $-.09 * *$ \\
\hline & {$[.02, .16]$} & {$[.09, .22]$} & {$[.01, .16]$} & {$[.05, .17]$} & {$[.00, .15]$} & {$[.03, .16]$} & {$[-.04, .11]$} & {$[-.15,-.02]$} \\
\hline \multirow[t]{2}{*}{ Political ideology } & $.16^{* *}$ & $.19 * *$ & $.11 * *$ & $.18 * *$ & $.10 * *$ & $.08 * *$ & -.03 & $-.09 * *$ \\
\hline & {$[.08, .23]$} & {$[.13, .25]$} & {$[.04, .18]$} & {$[.12, .24]$} & {$[.03, .18]$} & {$[.02, .14]$} & {$[-.10, .05]$} & {$[-.15,-.03]$} \\
\hline \multirow[t]{2}{*}{ Science knowledge } & $.15 * *$ & $.09 * *$ & $.18 * *$ & $.17 * *$ & $.15^{* *}$ & $.16^{* *}$ & $<.01$ & .04 \\
\hline & {$[.08, .22]$} & {$[.03, .15]$} & {$[.11, .25]$} & {$[.11, .23]$} & {$[.08, .22]$} & {$[.10, .22]$} & {$[-.08, .07]$} & {$[-.02, .10]$} \\
\hline \multirow[t]{2}{*}{ Science methodology } & .06 & -.04 & $.10^{*}$ & $.07 *$ & $.09 *$ & $.09 * *$ & -.02 & .05 \\
\hline & {$[-.02, .13]$} & {$[-.10, .02]$} & {$[.02, .17]$} & {$[.00, .13]$} & {$[.02, .17]$} & {$[.03, .15]$} & {$[-.09, .06]$} & {$[-.01, .11]$} \\
\hline \multirow[t]{2}{*}{ Religiosity } & -.04 & $.08 *$ & $<.01$ & $.19 * *$ & .02 & $.18 * *$ & $-.17 * *$ & $-.28 * *$ \\
\hline & {$[-.11, .04]$} & {$[.02, .14]$} & {$[-.08, .07]$} & {$[.13, .25]$} & {$[-.05, .09]$} & {$[.12, .24]$} & {$[-.24,-.10]$} & {$[-.34,-.23]$} \\
\hline \multirow[t]{2}{*}{ AOT-E } & .02 & $<.01$ & $.15^{* *}$ & $.11 * *$ & $.11 * *$ & $.17 * *$ & $<.01$ & $<.01$ \\
\hline & {$[-.05, .10]$} & {$[-.06, .06]$} & {$[.08, .22]$} & {$[.05, .17]$} & {$[.04, .18]$} & {$[.11, .23]$} & {$[-.08, .07]$} & {$[-.06, .06]$} \\
\hline \multirow[t]{2}{*}{ CRT } & $.16^{* *}$ & .06 & $.10 * *$ & .05 & $.12 * *$ & $.07 *$ & $.10 * *$ & $.08^{*}$ \\
\hline & {$[.08, .23]$} & {$[-.00, .12]$} & {$[.02, .17]$} & {$[-.01, .11]$} & {$[.05, .19]$} & {$[.00, .13]$} & {$[.02, .17]$} & {$[.02, .14]$} \\
\hline \multirow[t]{2}{*}{ BSR } & .01 & $.12 * *$ & -.05 & $.19 * *$ & .01 & $.13^{* *}$ & -.07 & $-.24 * *$ \\
\hline & {$[-.07, .08]$} & {$[.06, .18]$} & {$[-.12, .03]$} & {$[.13, .25]$} & {$[-.07, .08]$} & {$[.07, .19]$} & {$[-.15, .00]$} & {$[-.30,-.18]$} \\
\hline
\end{tabular}




\begin{tabular}{|c|c|c|c|c|c|c|c|c|}
\hline \multirow[t]{2}{*}{ Variable } & \multicolumn{2}{|c|}{ GMO safety } & \multicolumn{2}{|c|}{ Acupuncture rejection } & \multicolumn{2}{|c|}{ Essential oils rejection } & \multicolumn{2}{|c|}{ Detox rejection } \\
\hline & Lucid & Dynata & Lucid & Dynata & Lucid & Dynata & Lucid & Dynata \\
\hline \multirow[t]{2}{*}{ Political Partisanship } & .03 & -.01 & -.03 & -.04 & .02 & $-.11 * *$ & -.01 & -.05 \\
\hline & {$[-.05, .10]$} & {$[-.07, .05]$} & {$[-.11, .04]$} & {$[-.10, .02]$} & {$[-.05, .10]$} & {$[-.17,-.05]$} & {$[-.09, .06]$} & {$[-.12, .01]$} \\
\hline \multirow[t]{2}{*}{ Political ideology } & -.01 & $-.07 *$ & -.05 & -.04 & -.02 & $-.16^{* *}$ & -.06 & $-.10 * *$ \\
\hline & {$[-.08, .07]$} & {$[-.13,-.01]$} & {$[-.12, .02]$} & {$[-.11, .02]$} & {$[-.10, .05]$} & {$[-.22,-.10]$} & {$[-.14, .01]$} & {$[-.16,-.03]$} \\
\hline \multirow[t]{2}{*}{ Science knowledge } & $.23^{* *}$ & $.18^{* *}$ & .03 & -.02 & $.16^{* *}$ & $.17^{* *}$ & $.23 * *$ & $.21 * *$ \\
\hline & {$[.15, .30]$} & {$[.12, .24]$} & {$[-.05, .10]$} & {$[-.08, .04]$} & {$[.09, .23]$} & {$[.11, .23]$} & {$[.16, .30]$} & {$[.15, .26]$} \\
\hline \multirow[t]{2}{*}{ Science methodology } & $.18^{* *}$ & $.20 * *$ & .06 & .03 & $.14 * *$ & $.21 * *$ & $.18^{* *}$ & $.20 * *$ \\
\hline & {$[.10, .25]$} & {$[.14, .26]$} & {$[-.01, .13]$} & {$[-.03, .09]$} & {$[.06, .21]$} & {$[.15, .27]$} & {$[.10, .25]$} & {$[.14, .26]$} \\
\hline \multirow[t]{2}{*}{ Religiosity } & $-.22 * *$ & $-.25 * *$ & $-.19 * *$ & $-.26 * *$ & $-.23 * *$ & $-.36^{* *}$ & $-.23 * *$ & $-.33 * *$ \\
\hline & {$[-.29,-.14]$} & {$[-.31,-.19]$} & {$[-.26,-.12]$} & {$[-.32,-.21]$} & {$[-.30,-.16]$} & {$[-.41,-.31]$} & {$[-.30,-.15]$} & {$[-.38,-.27]$} \\
\hline \multirow[t]{2}{*}{ AOT-E } & $.15^{* *}$ & $.19^{* *}$ & .03 & -.06 & $.20 * *$ & $.23^{* *}$ & $.20 * *$ & $.18^{* *}$ \\
\hline & {$[.08, .22]$} & {$[.13, .25]$} & {$[-.04, .11]$} & {$[-.12, .00]$} & {$[.13, .27]$} & {$[.17, .29]$} & {$[.13, .27]$} & {$[.12, .24]$} \\
\hline \multirow[t]{2}{*}{ CRT } & $.26 * *$ & $.20 * *$ & $.13^{* *}$ & .06 & $.24 * *$ & $.21 * *$ & $.27 * *$ & $.25 * *$ \\
\hline & {$[.19, .33]$} & {$[.14, .26]$} & {$[.06, .20]$} & {$[-.01, .12]$} & {$[.16, .31]$} & {$[.15, .26]$} & {$[.20, .34]$} & {$[.19, .30]$} \\
\hline \multirow[t]{2}{*}{ BSR } & $-.20 * *$ & $-.32 * *$ & $-.13 * *$ & $-.30 * *$ & $-.25 * *$ & $-.43 * *$ & $-.21 * *$ & $-.43 * *$ \\
\hline & {$[-.27,-.13]$} & {$[-.38,-.27]$} & {$[-.20,-.05]$} & {$[-.36,-.24]$} & {$[-.32,-.18]$} & {$[-.47,-.37]$} & {$[-.28,-.13]$} & {$[-.48,-.38]$} \\
\hline
\end{tabular}




\section{What contributes more to beliefs about science: science knowledge or political}

ideology? Next, we directly compared whether science knowledge or political beliefs correlated more strongly with science beliefs. For this, we used the two versions of the pro-science beliefs scale: one where liberal beliefs accord with the pro-science position and one for conservative beliefs (this was not preregistered for Sample 1; see supplementary materials file 1 for details). To divide the items into partisan categories, we examined the correlations between political partisanship and each science topic, grouping the items together based on the direction of the correlation. This calculation allowed us to examine the overall correlation with ideology.

We then conducted a series of Fisher's $r$-to- $Z$ comparisons. Identical analyses on both samples revealed a consistent pattern of results. For issues where liberal ideology accords with the pro-science position (global warming, evolution, the big bang, and sex ambiguity), political ideology (Lucid: $Z=5.85, p_{\text {two-tailed }}<.001$; Dynata: $Z=12.04, p_{\text {two-tailed }}<.001$ ) and partisanship (Lucid: $Z=5.85, p_{\text {two-tailed }}<.001$; Dynata: $Z=12.04, p_{\text {two-tailed }}<.001$ ) were more strongly correlated with beliefs compared to science knowledge. For conservative pro-science issues (13 issues in total), the opposite pattern was evident: basic science knowledge was more strongly related to beliefs than political ideology (Lucid: $Z=3.52, p_{\text {two-tailed }}<.001$; Dynata: $Z=5.80, p_{\text {two- }}$ tailed $<.001$ ) and political partisanship (Lucid: $Z=2.57, p_{\text {two-tailed }}<.001$; Dynata: $Z=5.58, p_{\text {two- }}$ tailed $<.001)$.

What is the role of cognitive sophistication? The identity-protective cognition and analytic thinking accounts make competing predictions with respect to the association between cognitive sophistication and science beliefs. Specifically, the identity-protective cognition account predicts that people higher in cognitive sophistication are better able to interpret evidence in an ideology-consistent manner and therefore that (in the context of politicized issues) cognitive sophistication should correlate with attitudes in opposite directions for Democrats and 
Republicans. Under this model, we would expect to find clear instances where cognitive sophistication correlates in opposite directions for Democrats and Republicans. ${ }^{2}$ In contrast, the analytic thinking account simply predicts that people higher in cognitive sophistication would hold more (and stronger) pro-scientific beliefs. Under this model, we would expect to find that cognitive sophistication is associated with the pro-science position.

We simultaneously regressed each of the science belief scores onto a standardized composite of the cognitive sophistication variables $(\alpha=.71$; BSR was reverse-scored), political partisanship, and their interaction. Of the 17 science belief items, there were main effects of cognitive sophistication on all except for four issues: nuclear power, testosterone, IQ heritability, and gender differences on the SAT. There were also main effects of political partisanship on all except six issues: IQ heritability, homeopathy, GMOs, acupuncture, essential oils, and detoxing. More importantly, significant interactions were found for all except five topics: stereotypes, vaccines, homeopathy, GMOs, and detoxing (Tables S10-S26 report full details of each analyses in Sample 1, and Tables S27-S43 for Sample 2).

These results provide some partial support for identity-protective cognition. Critically, however, the identity-protective cognition account does not merely predict an interaction. Rather, the account predicts that cognitive sophistication facilitates identity-protective cognition, which means that partisans who are higher in cognitive sophistication will be more likely to hold the ideologically consistent position (the interaction emerges due to having both opposing partisans in the same sample; had we completed a study only among Republicans, the account would not predict a null correlation for a politicized issue). Thus, for an issue such as global warming, the

\footnotetext{
${ }^{2}$ The identity-protective cognition account argues specifically that cognitive sophistication increases belief when politically congenial - thus, a null correlation between cognitive sophistication and belief by itself (which would produce an interaction between cognitive sophistication and political ideology) should not constitute positive evidence for the account.
} 
prediction is a positive correlation between cognitive sophistication and belief among Democrats but a negative correlation among Republicans. Thus, cognitive sophistication should be significantly associated with science beliefs in opposite directions for Democrats relative to Republicans across a range of issues for the identity-protective cognition account to have broad support (see Pennycook et al., 2020 for more discussion of this issue). Therefore, for the issues for which there was a significant interaction, we separated Democrats and Republicans and investigated the correlations between cognitive sophistication and each issue independently (Table 3).

Notably, there was not a single issue in Sample 1 where cognitive sophistication correlated significantly in opposite directions for Democrats and Republicans, as would be expected under the identity-protective cognition account. As can be seen in Figure 2, partisans did not reason in different directions based on cognitive sophistication; the relative slopes for conservatives and liberals were remarkable similar and not contingent on the belief that a topic was politically divisive (also see Figure S2). Instead, for the 12 topics on which an interaction was observed, cognitive sophistication is apparently important for one party but not the other. This indicates that there may be countervailing forces that dull the positive impact of cognitive sophistication on pro-scientific belief in some cases (consistent with politically motivated reasoning broadly), but it does not indicate that cognitive sophistication exacerbates political biases (as is the central claim of the identity-protective cognition account).

In Sample 2, we did observe divergent correlations for three issues: belief in global warming, the big bang, and evolution. These topics are often focused on when investigating identity-protective cognition (Drummond \& Fischhoff, 2017b; Kahan, 2013; Kahan et al., 2012), perhaps because of their extreme divisiveness along political and religious boundaries. At any rate, the pattern of results for the remaining topics does not support the identity-protective 
cognition account. This implies that it may not be the case that people generally use explicit reasoning (bolstered by cognitive sophistication) in a motivated way when it comes to contentious scientific issues - at least, absent direct evidence within the context of these specific issues (see Studies 2 and 3). Furthermore, there were issues that were both considered to be similarly politically divisive in our pretest (see Figure 2) and that were correlated with political ideology at similar levels (such as the gender wage gap question) but that did not produce this pattern of results. Indeed, the only issue where cognitive sophistication consistently and significantly predicted the ostensibly anti-science belief related to re-transitioning genders, and this was true among both Democrats and Republicans in the Dynata sample (for further on why this issue is an exception, see below). To summarize, across 17 issues and separating Democrats and Republicans across both samples, cognitive sophistication was significantly positively associated with pro-science beliefs in 40 out of 68 possible cases, was not correlated with beliefs in 22 cases, and was significantly negatively correlated with beliefs in 6 cases. Removing the outlying item about re-transitioning genders modifies this to positive in 40 out of 64 cases, null in 21 , and negative in 3 cases. Thus, regardless of partisanship, cognitive sophistication is broadly associated with pro-scientific beliefs across issues and only rarely associated with antiscience beliefs. 
Figure 2. Depiction of interactions between political affiliation and cognition sophistication arranged by belief that the topic is politically divisive. Data are from Sample 2 (Dynata, $N=1,018$ ); data are arranged according to the belief that the topics are politically divisive as rated by an independent sample $(N=203)$; $\mathrm{x}$-axis = standardized cognitive sophistication; $\mathrm{y}$-axis = probability of agreeing with pro-science position; shaded envelope indicates $95 \%$ CI; the full set of graphs for Sample 2 are depicted in the supplementary materials.

elief that topic is

politically divisive $\mathbf{7 2} \%$

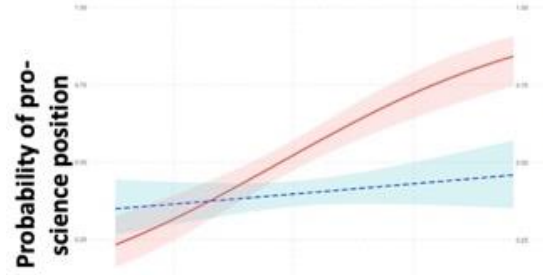

Wage gap
$71 \%$

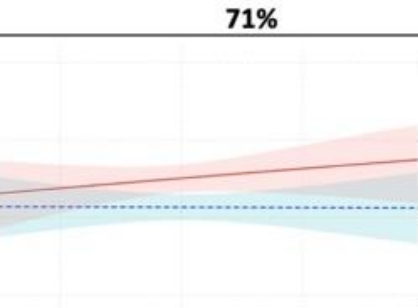

Nuclear Power
$70 \%$

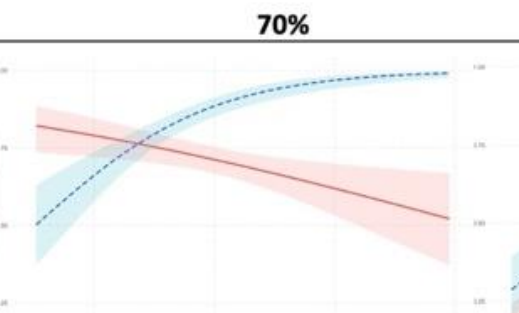

Global Warming
$52 \%$

Conservatives (Republicans)
$60 \%$

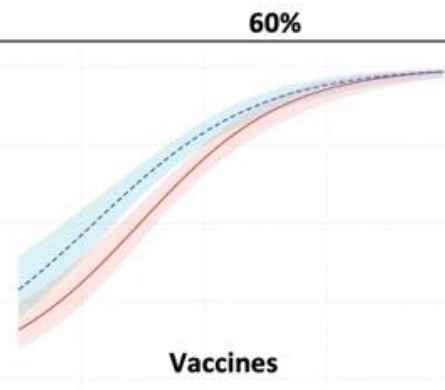


Table 3. Correlations between cognitive sophistication and pro-science issues for democrats and republicans in Samples 1 and 2. Values in brackets indicate 95\% CI. 'Indicates $p<.05$ for the interaction between political affiliation and cognitive sophistication in Sample 1 and ${ }^{ \pm}$in Sample 2; ${ }^{*} p<$ $.05 . * * p<.01$.

\begin{tabular}{|c|c|c|c|c|}
\hline \multirow{3}{*}{ Pro-science belief } & \multicolumn{2}{|c|}{$\begin{array}{l}\text { Sample 1: Lucid } \\
\quad(N=691)\end{array}$} & \multicolumn{2}{|c|}{$\begin{array}{c}\text { Sample 2: Dynata } \\
(N=1,018)\end{array}$} \\
\hline & Democrats & Republicans & Democrats & Republicans \\
\hline & $r$ & $r$ & $r$ & $r$ \\
\hline \multirow[t]{2}{*}{ 1. Global warming belief ${ }^{ \pm}$} & $.30^{* *}$ & -.04 & $.40^{* * *}$ & $-.16^{* *}$ \\
\hline & {$[.21, .39]$} & {$[-.15, .07]$} & {$[.33, .47]$} & {$[-.25,-.07]$} \\
\hline \multirow[t]{2}{*}{ 2. Wage gap sexism ${ }^{\dagger \pm}$} & -.00 & $.22 * *$ & .02 & $.32 * *$ \\
\hline & {$[-.11, .10]$} & {$[.11, .32]$} & {$[-.07, .10]$} & {$[.23, .40]$} \\
\hline \multirow[t]{2}{*}{ 3. Sex ambiguity ${ }^{\dagger \pm}$} & $.37 * *$ & -.02 & $.29 * *$ & .04 \\
\hline & {$[.28, .46]$} & {$[-.13, .09]$} & {$[.21, .37]$} & {$[-.06, .13]$} \\
\hline \multirow[t]{2}{*}{ 4. Evolution belief ${ }^{\dagger \pm}$} & $.14 * *$ & .01 & $.27 * *$ & $-.15^{* *}$ \\
\hline & {$[.04, .24]$} & {$[-.10, .12]$} & {$[.19, .35]$} & {$[-.24,-.06]$} \\
\hline \multirow[t]{2}{*}{ 5. Big bang belief ${ }^{\dagger \pm}$} & $.20 * *$ & .02 & $.21 * *$ & $-.21 * *$ \\
\hline & {$[.10, .30]$} & {$[-.09, .13]$} & {$[.13, .29]$} & {$[-.30,-.12]$} \\
\hline \multirow[t]{2}{*}{ 6. Stereotype accuracy ${ }^{ \pm}$} & $.21 * *$ & $.29 * *$ & $.23 * *$ & $.37 * *$ \\
\hline & {$[.11, .31]$} & {$[.18, .39]$} & {$[.15, .31]$} & {$[.29, .45]$} \\
\hline \multirow[t]{2}{*}{ 7. Vaccine acceptance ${ }^{ \pm}$} & $.37 * *$ & $.31 * *$ & $.46 * *$ & $.48^{* *}$ \\
\hline & {$[.28, .46]$} & {$[.20, .40]$} & {$[.39, .52]$} & {$[.41, .55]$} \\
\hline \multirow[t]{2}{*}{ 8. Re-transitioning genders ${ }^{\dagger}$} & $-.16 * *$ & .07 & $-.16^{* *}$ & $-.19 * *$ \\
\hline & {$[-.26,-.06]$} & {$[-.04, .18]$} & {$[-.24,-.07]$} & {$[-.27,-.10]$} \\
\hline \multirow[t]{2}{*}{ 9. Nuclear power safety ${ }^{\dagger}$} & .05 & $.19 * *$ & -.00 & .00 \\
\hline & {$[-.06, .15]$} & {$[.08, .29]$} & {$[-.09, .08]$} & {$[-.09, .09]$} \\
\hline \multirow[t]{2}{*}{ 10. Testosterone athleticism ${ }^{\dagger}$} & .07 & $.28 * *$ & .07 & .06 \\
\hline & {$[-.04, .17]$} & {$[.18, .38]$} & {$[-.02, .15]$} & {$[-.03, .15]$} \\
\hline \multirow[t]{2}{*}{ 11. IQ heritability ${ }^{\dagger \pm}$} & .07 & $.21 * *$ & $.16^{* *}$ & .01 \\
\hline & {$[-.03, .17]$} & {$[.10, .31]$} & {$[.08, .25]$} & {$[-.08, .10]$} \\
\hline \multirow[t]{2}{*}{ 12. Homeopathy rejection } & $.14 * *$ & -.08 & $.12 * *$ & $.13 * *$ \\
\hline & {$[.04, .24]$} & {$[-.18, .03]$} & {$[.03, .20]$} & {$[.03, .22]$} \\
\hline \multirow[t]{2}{*}{ 13. GMO acceptance } & $.35^{* *}$ & $.23 * *$ & $.31 * *$ & $.33 * *$ \\
\hline & {$[.25, .44]$} & {$[.13, .34]$} & {$[.23, .38]$} & {$[.24, .41]$} \\
\hline \multirow[t]{2}{*}{ 14. Acupuncture rejection ${ }^{\dagger \pm}$} & $.21 * *$ & -.00 & .04 & $.15^{* *}$ \\
\hline & {$[.11, .31]$} & {$[-.11, .11]$} & {$[-.05, .12]$} & {$[.06, .24]$} \\
\hline \multirow[t]{2}{*}{ 15. Essential oil rejection ${ }^{\dagger}$} & $.37 * *$ & $.20 * *$ & $.37 * *$ & $.32 * *$ \\
\hline & {$[.28, .45]$} & {$[.09, .30]$} & {$[.30, .44]$} & {$[.24, .40]$} \\
\hline \multirow[t]{2}{*}{ 16. Detox rejection } & $.40 * *$ & $.23 * *$ & $.34 * *$ & $.38 * *$ \\
\hline & {$[.31, .49]$} & {$[.12, .33]$} & {$[.27, .41]$} & {$[.30, .46]$} \\
\hline \multirow[t]{2}{*}{ 17. SAT gender differences ${ }^{\dagger \pm}$} & -.00 & .04 & .02 & $.15^{* *}$ \\
\hline & {$[-.11, .10]$} & {$[-.07, .15]$} & {$[-.07, .10]$} & {$[.06, .24]$} \\
\hline
\end{tabular}




\section{Study 2}

Study 1 found surprisingly inconsistent correlations between political partisanship and science beliefs across a wide range of issues. Furthermore, cognitive sophistication was consistently positively correlated with pro-science beliefs across issues. There were, however, a number of cases where political partisanship interacted with cognitive sophistication, which is broadly consistent with politically motivated reasoning effects. Nonetheless, the vast majority of these cases where characterized simply by weaker (or null) correlations between cognitive sophistication and the science belief in question, indicating weak evidence for the claim that cognitive sophistication increases belief in anti-science beliefs for contentious topics (as follows from the identity-protective cognition account).

Notably, and consistent with past work (Kahan et al., 2012), global warming was quite strongly correlated with political ideology/partisanship - and, indeed, what one of two issues (along with sex ambiguity) where ideology/partisanship was correlated above $r>.30$ in both samples. Importantly, in at least in one of the two samples, cognitive sophistication was negatively correlated with global warming belief among Republicans. This indicates that, although identity-protective cognition may not strongly explain most science beliefs, it may nonetheless be a good way to characterize the underlying mechanisms for some select scientific issues.

To investigate this issue more directly, we adapted an experimental paradigm that has been used in past work (Bolsen et al., 2014) to increase political motivations and focused specifically on an issue for which there was the strongest evidence for political divisiveness: global warming. Specifically, participants were given a set of arguments that argue for or against anthropogenic climate change and asked explicitly to "view the information through a political 
lens" - a manipulation that intended to parallel what is assumed to occur under politically motivated reasoning for scientific issues. Critically, inducing this motivation among our participants allows us to test whether increasing political motivations interacts with cognitive sophistication. The identity-protective cognition account predicts that polarization around climate change should increase more strongly given increased political motivations, particularly among individuals who are higher in cognitive sophistication. This therefore allows us to test for experimental evidence of identity-protective cognition in a particularly charged context, which would help inform our interpretation of Study 1.

\section{Method}

\section{Participants}

In total, 1176 participants started the experiment. Of this, 126 participants did not give a correct response to the first attention check question and therefore could not take part in the experiment (hence, produced no data). We included all data from participants who made it to this point. In the control condition, 524 participants took part (120 female, 318 male and 2 others, Mean age $=51.6$ years, $\mathrm{SD}=18.2$ years), 227 identified as Democrat and 208 identified as Republican. In the "motivated" condition, 525 participants took part (125 female, 291 male, Mean age $=52.6$ years, $\mathrm{SD}=18.3$ years), 202 people identified as a Democrat and 214 identified as a Republican.

\section{Materials}

Climate Change arguments. Participants received 6 arguments about climate change. All arguments were pre-tested and taken from Bago et al. (2020). Half of these arguments were supporting arguments for anthropogenic climate change. These were scientific arguments, mostly explaining how climate change affects our environment, or how human activity causes climate 
change, all related to climate change, while the other half of the arguments argued against that humans causing climate change or that it would have bad consequences. All of these arguments were presented in a non-partisan manner, there were no party cues, or politicians mentioned whatsoever. Arguments were content-counterbalanced, and participants never saw both the pro and contra version of a given content. That is, participants were presented with only one of the two versions of one argument, but were always presented with 3 pro and 3 contra arguments (out of an item pool of 10 arguments). After each argument, we asked people "How much do you agree with this argument? ( $0=$ completely disagree, $100=$ completely agree)". Participants could give a response on a slider. To make sure that the position of the handler on the slide does not bias participants, the handler was not shown until participants first clicked on the slide. For the analysis, for every participant, we categorized arguments based on its concordance to one's own political partisan identity: contra arguments were "concordant" for Republicans and "discordant" for Democrats, while pro arguments were "discordant" to Republicans and "concordant" to Democrats.

Experimental Manipulation. To make partisanship salient, in the "motivated" condition we presented people with the following instructions:

"When thinking about the argument, please try to view the information through a political lens. This will help you put the argument in the context of the political divide that surrounds the issue. We will later ask you about your party and why you affiliate with it (or why you choose to not affiliate with a party)."

Similar manipulations have been shown to be effective in making people focus on the partisanship while evaluating new information (Bolsen et al., 2014). Participants were shown 
these instructions at the beginning of the experiment (after the general introduction, see below) and on the top of the screen with every argument.

Procedure. First participants were presented with an ethical consent form, followed by two pre-treatment attention check questions. After they were presented with the general instructions of the study (instructions were kept the same among conditions):

"Welcome to this experiment!

In this experiment, you will be presented with different arguments regarding climate change and its potential effects. You will be asked to indicate how much you agree with the argument on a scale from $0=$ completely disagree to $100=$ completely agree. Please carefully read through the arguments before responding.

You will be presented with 6 arguments. The experiment will take about 15 minutes to complete, and will demand your full attention.

Before you would receive the arguments, we will ask you some questions concerning, science, technology and health.

Press 'Next' to continue!"

Next, participants received the same belief in science questionnaire as in Study 1 (17 statements about science altogether). After, participants in the "motivated" condition received the partisanship saliency instructions, and then they were presented with the partisanship arguments in a randomized order.

At the end of the experiment, participants were asked to answer a 6-item version of the Cognitive Reflection Test then a measure of knowledge of science (from Study 1). In the end, they were presented with a number of questions assessing various demographics and political preferences. Most importantly, to measure political preference, we asked participants "Which of 
the following best describes your political preference? 'Strongly Democratic/Democratic/Lean Democratic/Lean Republican/Republican/Strongly Republican"', which measure we used to categorize people into "Democrats" and "Republicans" (which was necessary to be able to categorize items into "concordant" and "discordant" categories).

Exclusions. As preregistered, we excluded all participants who did not correctly solve the second attention screening question (although they were allowed to participate in the experiment). In total, 241 people were excluded (120 from the control and 121 from the motivation condition), and 808 were further analyzed.

Statistical analysis. We used linear mixed effect models and the lmerTest package in R. We deviated from the preregistration to follow best practice recommendations in the field (Barr et al., 2013), which is to try to fit the maximal model (i.e., begin by adding all possible random slopes and removing terms until the model converges and has no singularity issues). Specifically, we preregistered that we would use random intercepts (and if it converges, random slopes of item contents), but not random slopes for our fixed effects. However, this was suboptimal as the maximal model procedure has been argued to decrease false positive errors (Barr et al., 2013). We therefore ran the more conservative model, in which we allowed the random intercept of subjects to vary over argument consistency, and the random intercept of argument content over consistency and cognitive sophistication score. As fixed effects, we added concordance (coded as: 0.5 concordant, -0.5 discordant $)$, experimental condition $(0-$ control, $1-$ motivated $)$ and continuous cognitive sophistication score (the average score on the CRT of each participant). The preregistered analysis is presented in the supplementary materials.

\section{Results}


Figure 3 summarizes the results. First - replicating the pattern for belief in Study 1 using our climate change arguments - we found a significant interaction between cognitive sophistication and concordance, $b=25.2,95 \% \mathrm{CI}=[12.2,38.3], p<.001$, such that more cognitively sophisticated individuals were more likely to agree with concordant than discordant information (i.e., there was an association between sophistication and polarization). We did not find a significant two-way interaction between the political motivation manipulation and concordance, $b=5.7,95 \% \mathrm{CI}=[-3.5,14.9], p=.227$; however, we found a marginally significant three-way interaction between political motivation, concordance, and cognitive sophistication, $b=-16.5,95 \% \mathrm{CI}=[-35.2,2.1], p=.082$ (this three-way interaction was significant in the preregistered analysis plan and there was moderate evidence for it using a preregistered Bayesian analysis; see Supplementary Materials file 1). Interestingly, the negative beta suggests that there was a tendency toward the opposite direction than was predicted by the identity-protective cognition account: Participants who scored lower on our cognitively sophistication measure were, if anything, the ones who were more divided about climate change in the political motivation condition relative to the control condition. As can be seen in Figure 3, the trend for the individuals who are higher in cognitive sophistication is for them to be less polarized in the political motivation condition. 


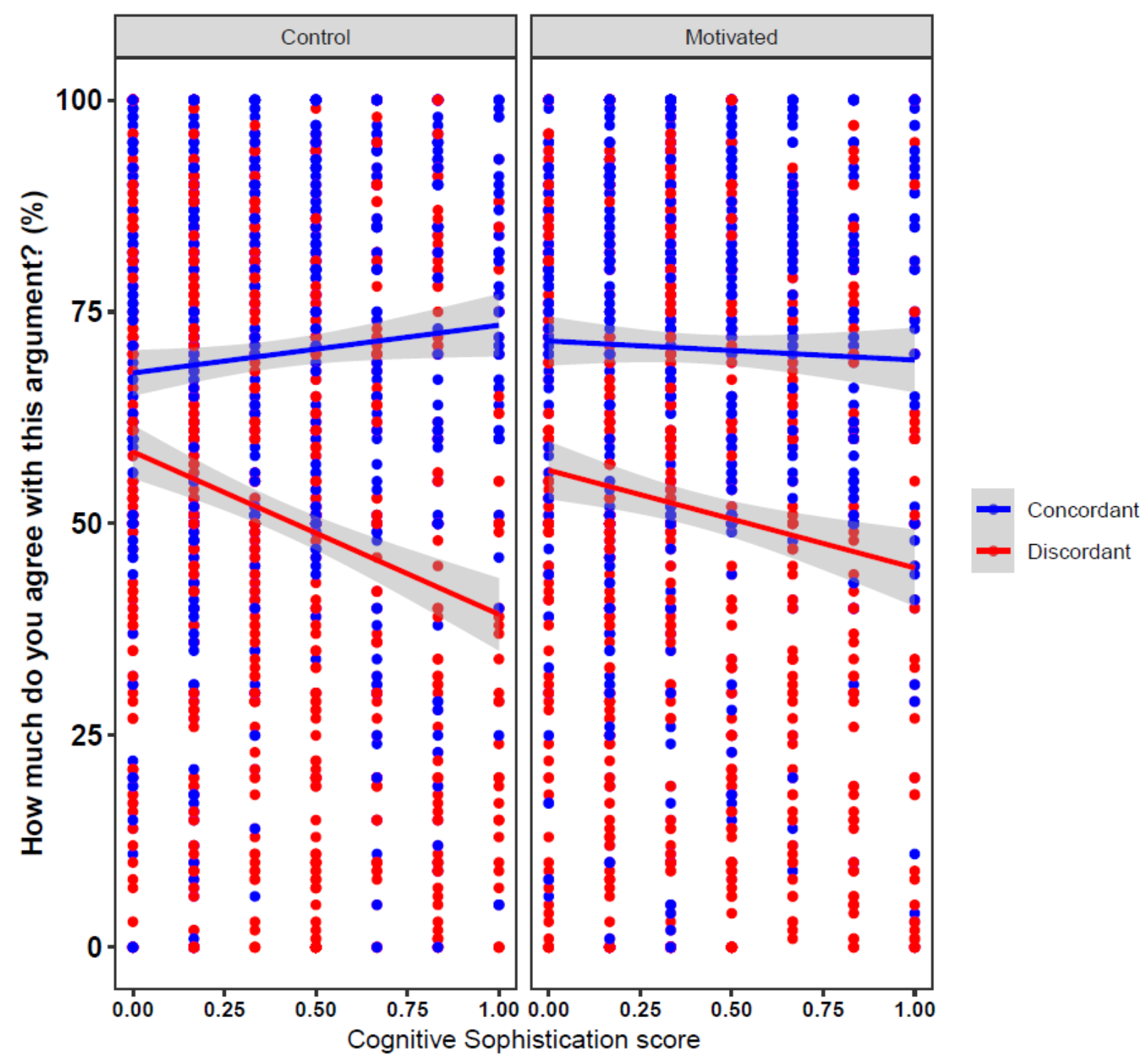

Figure 3. Agreement scores on the climate change arguments (Study 2) as a function of cognitive sophistication and concordance, separately for the control and motivated reasoning experimental groups. Error bars are 95\% CI intervals.

For completeness, we also report the other effects in the model. We found a main effect of concordance, $b=9.3,95 \% \mathrm{CI}=[2.5,16], p<.001$, such that people prefer concordant over discordant information (as would be expected). We also found a main effect of cognitive sophistication score, $b=-7,95 \% \mathrm{CI}=[-13.3,-0.7], p=.031$, such that more cognitively sophisticated individuals had weaker overall agreement with the arguments. There was no main 
effect of condition, $b=0.7,95 \% \mathrm{CI}=[-3.4,5], p=.730$, and no interaction between cognitive sophistication and condition, $b=0.44,95 \% \mathrm{CI}=[-7.9,8.8], p=.917$.

\section{Study 3}

Study 2 produced a surprising trend: Not only was cognitive sophistication not associated with increased polarization when participants were asked to think about the information through a partisan lens, but the pattern emerged as the polar opposite. This, however, was marginally significant. In Study 3, we set out to replicate Study 2 with one small change to the manipulation that was intended to increase partisan motivations. In particular, we removed the following sentence from the instructions: "This will help you put the argument in the context of the political divide that surrounds the issue." We thought that this may be interpreted (perhaps more so by individuals who are higher in cognitive sophistication) as a prescription to avoid the political divide. Hence, the participants were simply instructed to do the following in the partisan motivation condition: "When thinking about the argument, please try to view the information through a political lens. We will later ask you about your party and why you affiliate with it (or why you choose to not affiliate with a party)."

\section{Method}

\section{Participants}

In total, 1404 participants started the experiment. Of this, 196 participants did not give a correct response to the first attention check question and therefore could not take part in the experiment (hence, produced no data). We included all data from participants who made it to this point. In the control condition, 567 participants took part (264 female, 238 male and 2 others, Mean age $=45.6$ years, $\mathrm{SD}=17.6$ years $), 341$ identified as Democrat and 246 identified as Republican. In the "motivated" condition, 577 participants took part (232 female, 231 male and 3 
others, Mean age $=46.4$ years, $\mathrm{SD}=28.6$ years), 351 people identified as a Democrat and 226 identified as a Republican.

\section{Materials \& Procedure}

The same materials were used as in Study 2. We changed only a few things about the experiments compared to Study 2. First, we changed the instructions manipulation to the following:

"When thinking about the argument, please try to view the information through a political lens. We will later ask you about your party and why you affiliate with it (or why you choose to not affiliate with a party)."

Second, we measured political partisanship before the actual arguments and instructions were presented to make sure responding to that question was unaffected by the manipulation and the arguments. Third, after the climate change experiment, we presented participants with an additional question regarding their opinion about the 2007 Energy Act (Bolsen et al., 2014). This is what we asked exactly:

"We are next going to ask you what you think about parts of the 2007 Energy

Independence Act.

[Instructions for partisan motivation in the "motivated" condition; no other instructions in the control condition]

The Act included the following provisions:

- Requires U.S. automakers to boost gas mileage to 35 miles per gallon for all passenger cars by 2020 , which is a $40 \%$ increase.

- Funds for research and development of solar and geothermal energy, and for the 
increased production of biofuels.

- Provides small businesses loans toward energy efficiency improvements

Given this information, to what extent do you oppose or support the Energy Act? (0completely oppose, 100-completely support)"

We included this as a conceptual replication of Bolsen et al. (2014) who they showed that once participants are instructed to think about their partisan motivation, the gap between Democrats and Republicans for the support of the 2007 Energy Act was increased (compared to a control condition).

Exclusion. As in Study 2, we excluded all participants, who did not give a correct question to the second attention check question (although they were allowed to participate in the experiment). In total, 268 people were excluded (133 from the control and 135 from the motivated condition), and 911 were further analysed.

\section{Results}

Figure 4 summarizes the findings for the climate change arguments. We used the same analysis strategy as in Study 2: we allowed the random intercept of subjects to vary over argument consistency, and the random intercept of argument content over consistency and cognitive sophistication score (note that we also deviated from our preregistered analysis plan in Study 3 as we did in Study 2; see the Supplementary Materials file 1 for the preregistered analyses). Replicating Studies 1 and 2, there was a two-way interaction between cognitive sophistication and argument consistency, $b=31.99,95 \% \mathrm{CI}=[20.6,43.4], p<.001$, such that the gap between concordant and discordant arguments was greater for more cognitively sophisticated individuals. There was also an interaction between experimental condition and consistency, $b=9.3,95 \% \mathrm{CI}=[1.6,17], p=.017$, such that the overall level of political 
polarization was greater in the partisan motivation condition relative to the control. Critically, there was also a three-way interaction between cognitive sophistication, the political motivation condition, and consistency, $b=-26.7,95 \% \mathrm{CI}=[-43.8,-9.7], p=.002$, in the same direction as in Study 2 (we also found strong evidence for this interaction using a preregistered Bayesian analysis, see Supplementary Materials file 1). Specifically, participants who were higher in cognitive sophistication were, if anything, less politically polarized in the motivation manipulation (whereas those lower in cognitive sophistication were more polarized). Put differently, the political motivation manipulation decreased the effect of cognitive sophistication on the gap between politically consistent and inconsistent arguments. 


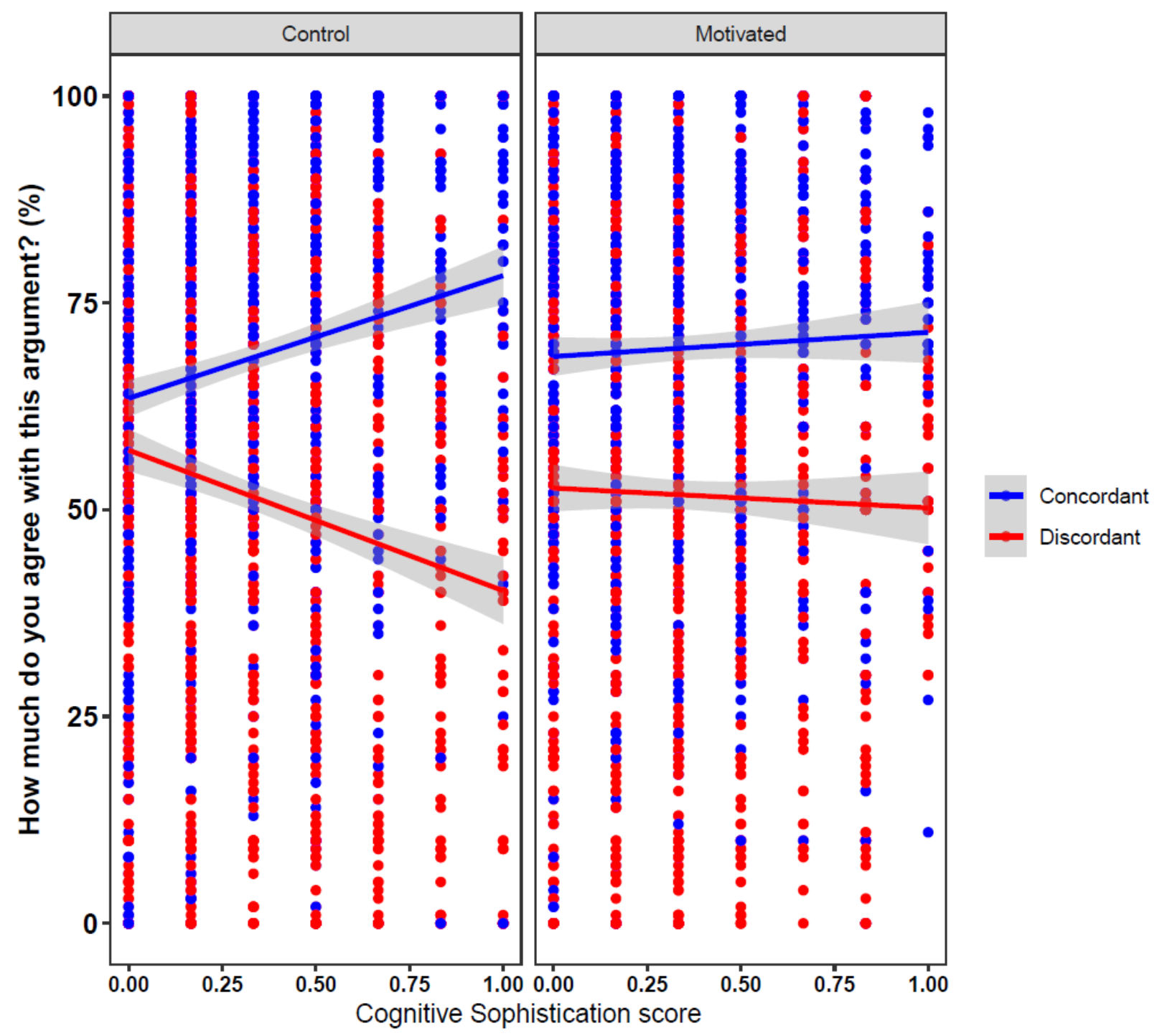

Figure 4. Agreement scores on the climate change arguments (Study 3) as a function of cognitive sophistication and concordance, separately for the control and motivated reasoning experimental groups. Error bars are 95\% CI intervals.

We also found a main effect of argument consistency, $b=6.3,95 \% \mathrm{CI}=[0.79,11.8], p=$ .026 , indicating that people agreed with concordant arguments more than with discordant arguments. However, there were no main effects of cognitive sophistication, $b=-0.95,95 \% \mathrm{CI}=$ $[-6.4,4.4], p=.730$, nor condition, $b=-0.005,95 \% \mathrm{CI}=[-3.4,3.4], p=.998$, and there was also no two-way interaction between cognitive sophistication score and experimental condition, $b=$ $1.7,95 \% \mathrm{CI}=[-5.9,9.3], p=.664$. 
We also analyzed the responses on the Energy Act question, but instead of using "argument consistency" in the model, we simply added partisanship (Democrats - -0.5 , Republicans, 0.5). Overall, we replicated the patters we observed with regard to the climate change arguments (see Figure 5 for graphical representation). We found a significant two-way interaction between partisanship and cognitive sophistication score, $b=26.9,95 \% \mathrm{CI}=[10.9$, 43], $p=.001$, and a three-way interaction between experimental condition, partisanship and cognitive sophistication score, $b=-26.9,95 \% \mathrm{CI}=[-51,-2.8], p=.029$. No other interaction or main effect were significant (although two were marginally significant): no interaction between cognitive sophistication score and condition, $b=-1.5,95 \% \mathrm{CI}=[-13.6,10.5], p=.805$, a marginally significant interaction between condition and partisanship, $b=9.9,95 \% \mathrm{CI}=[-0.96$, 20.7], $p=.074$, no main effect of cognitive sophistication, $b=5.3,95 \% \mathrm{CI}=[-2.7,13.4], p=$ .193, a marginally significant main effect of partisanship, $b=6.6,95 \% \mathrm{CI}=[-0.7,14], p=.076$, and no main effect of condition, $b=0.75,95 \% \mathrm{CI}=[-4.7,6.2], p=.786$. 


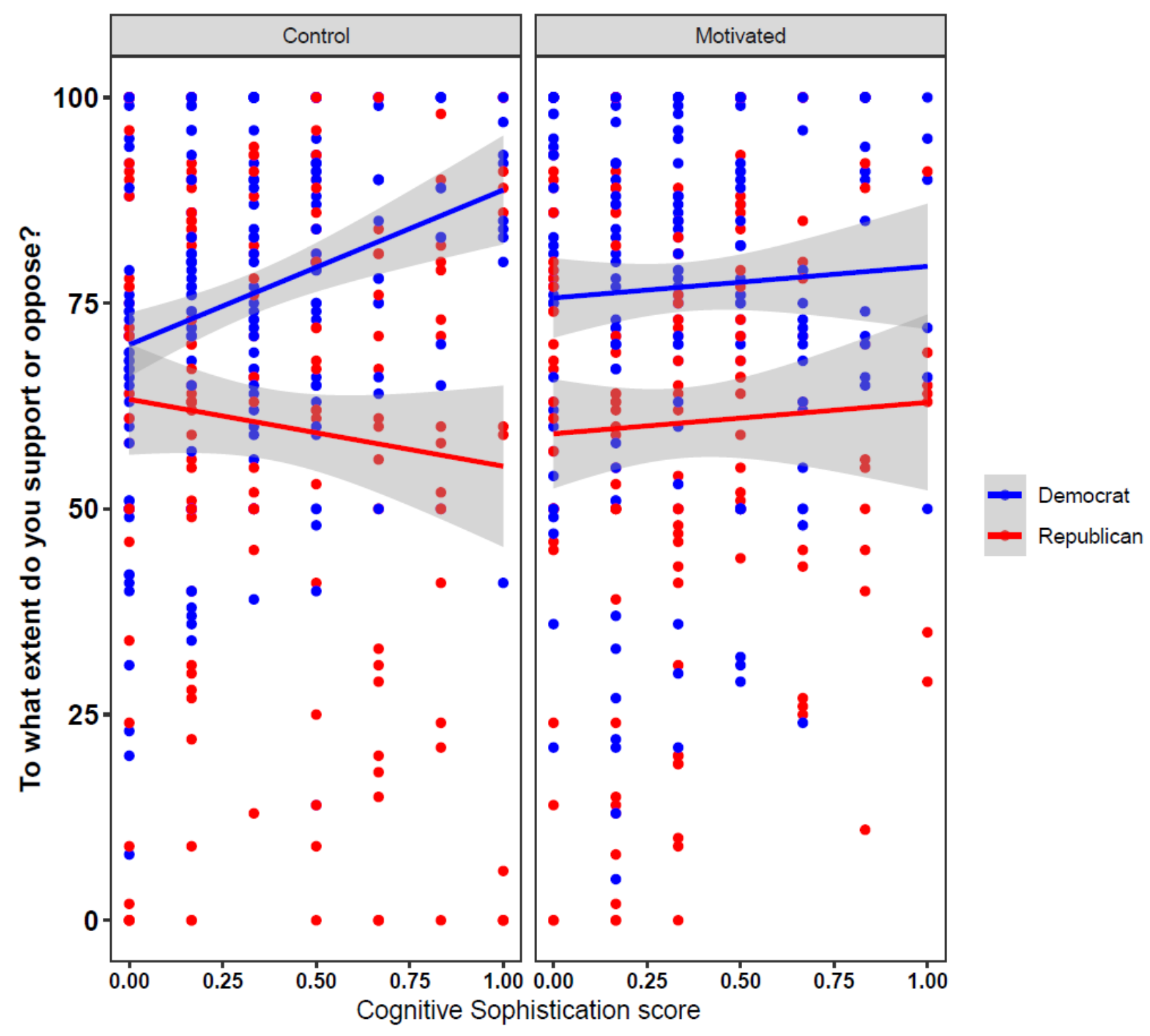

Figure 5. Responses on the 2007 Energy Act question as a function of cognitive sophistication and concordance, separately for the control and motivated reasoning experimental groups. Error bars are $95 \%$ CI intervals.

\section{General Discussion}

We draw three main conclusions from these results. First, although political ideology and partisanship were broadly correlated with beliefs about science, these correlations were only large $(r$ 's $>.30)$ for a minority of scientific issues. Basic science knowledge and cognitive sophistication were as correlated with science beliefs as ideology and, in many cases, were more strong and consistent predictors. Second, we found relatively weak evidence for the identity- 
protective cognition account, both via correlational tests across a large range of controversial scientific issues as well in two experiments where political motivation was induced. Specifically, there was not a single case across the 17 issues that we investigated where cognitive sophistication was consistently associated in opposite directions for Democrats versus Republicans across both samples (although there were 3 such cases in one of the two samples). Instead, cognitive sophisticated tended to be positively associated with pro-science belief across issues regardless of political partisanship. Furthermore, having participants evaluate arguments about global warming (one of the issues where there was some evidence for identity-protective cognition) in a politically motivated way actually decreased polarization among cognitively sophisticated individuals. This indicates that the effect of political motivations on scientific evidence evaluation may not be as straightforward as is broadly contended.

The role of politically motivated reasoning in science beliefs. Consistent with the politically motivated reasoning perspective, we found that ideology was generally correlated with science beliefs. However, these correlations were perhaps weaker than one might expect. For example, in Sample 1 of Study 1, there were only 5 (out of 17) issues for which political ideology had a strong or medium $(r>.20)$ correlation with belief (global warming, big bang, evolution, sex ambiguity, gender wage gap), and two of them (big bang and evolution) were more strongly correlated with religious belief. In Sample 2 of Study 1 there were only 3 issues (global warming, sex ambiguity, and gender transitions) with a strong or medium correlation. Interestingly, for the remainder of the issues (when aggregated), basic science knowledge was a stronger overall predictor that political ideology. This is notable because we deliberately selected issues that were ostensibly partisan (and a separate sample of participants also believed them to be so; see Table S2). Thus, the failure of political ideology to be the strongest and most 
consistent correlate of people's beliefs about a variety of topics may come as a surprise given the apparent importance of politically motivated reasoning in the literature. To be clear, however, there is no question that science is often political - this is evidenced, for example, by numerous scientists working as lobbyists and advising government policymakers (Scheufele, 2013, 2014). Rather, our evidence indicates that politicization (Gauchat, 2012; Scheufele, 2014) does not apparently spill over (to a great extent) into more than just a few select issues.

One criticism of our approach, however, is that we only measured political ideology and partisanship, but did not assess political engagement or sophistication (Abramowitz, 2010; Prior, 2013; Vitriol et al., 2020). Models of public opinion change have long noted that one's attentiveness to political issues will be strongly related to one's political motivations (Zaller, 1992), and therefore that politically motivated reasoning will be greatest among those who are the most politically sophisticated (Taber \& Lodge, 2006). Our results speak to the predictive validity of political ideology/partisanship in the aggregate, but it is possible (if not probable) that ideology plays a stronger role across various scientific issues for specific segments of the population (such as the highly politically engaged). Indeed, it is unlikely that science-related information would trigger politically motivated reasoning among people who are not sufficiently engaged with politics to know how they should react to the information based on their political allegiances. Thus, our data do not rule out a potentially important role of politically motivated reasoning for some people and among some scientific issues. Still, an alternative (and plausible) pattern of results would have indicated strong correlations between political ideology and science beliefs across numerous issues, and this clearly would have expanded the purview and importance of politically motivated reasoning - we do not find strong evidence for this, however. Nonetheless, future research on science beliefs should measure political engagement and 
sophistication directly and contrast this with the effects of cognitive sophistication to investigate the potential role of politically motivated reasoning more directly than we have done here.

Weak evidence for identity-protective cognition. A critical prediction derived from the identity-protective cognition account is that partisans use deliberative ("System 2") reasoning to interpret evidence that is politically consistent and, therefore, that people who are higher in cognitive sophistication should be more likely to believe (or disbelieve) in science when it is consistent (or inconsistent) with their political ideology (Kahan, 2013; Kahan et al., 2012; Mccright \& Dunlap, 2011). However, we found no correlational evidence of this in Sample 1 of Study 1 and only found evidence in Sample 2 of Study 1 on three issues: belief in global warming, the big bang, and evolution. Thus, we found positive evidence for identity-protective cognition in only 3 out of 34 possible tests across both samples. These issues are among the most commonly investigated science beliefs in the literature, and thus it can be seen how restricting focus to these few hot-button issues could lead to a distorted view of the underlying mechanisms that lead to anti-science belief.

While neither the politically motivated reasoning nor identity-protective cognition accounts inherently make claims about the broad-ness or narrow-ness of their predictive utility, it is important to determine whether their predictions generalize broadly across scientific issues. Although the evidence for a broad overriding impact of political ideology across issues was fairly weak, we did find a number of interactions between political partisanship and cognitive sophistication, which can be taken as broad evidence for identity-protective cognition. Importantly, though, examination of the interactions revealed that (in most cases) cognitive sophistication was simply uncorrelated with pro-science beliefs for one group but significantly correlated for the other (see Table 3). Such a result, in our view, should not be considered more 
than weak evidence for politically motivated reasoning since the provenance of null correlations are surely multitudinous.

Indeed, null correlations between cognitive sophistication and science beliefs occurred for issues that partisans did not disagree about. For example, consider the case of acupuncture, where cognitive sophistication was associated with scepticism among Democrats (but not Republicans) in Sample 1 and among Republicans (but not Democrats) in Sample 2. And yet, there was also no significant overall correlation between political ideology or partisanship and beliefs about the scientific basis of acupuncture. If a mere interaction between cognitive sophistication and ideology were sufficient evidence for identity-protective cognition, one would need to contend that there is evidence in a case such as acupuncture, where partisan not only don't disagree, but that the underlying nature of the interaction reversed across samples. Thus, as with many other issues, the most parsimonious explanation is that there is (an albeit small and potentially fleeting) association between cognitive sophistication and pro-scientific beliefs about acupuncture and that political ideology is not particularly relevant.

A potential criticism of our approach is that we investigated a set of science beliefs that have not been subject to political polarization and hence it is no surprise that political ideology was not a consistent predictor across issues. But how else could one know if political ideology is relevant for a specific issue than to investigate it? Certainly, none of the issues we investigated were innocuous and, as noted, lay people thought each issue was likely to be politically contentious (although, of course, this varied substantially across issues). Furthermore, there was a great deal of disagreement across issues (see Figure 1) and partisans did, generally, disagree about most issues - however, these differences were plainly smaller, in our view, than would be expected given the past literature and layperson estimates. 
Focusing specifically on global warming - perhaps the most contentious scientific issue (e.g., the correlation between political partisanship and belief in anthropogenic climate change was $r=-.45$ in Sample 1 of Study 1) - we found no evidence that inducing political motivations caused individuals who are higher in cognitive sophistication to become more polarized. In fact, across two highly-powered preregistered experiment, we found that having people consider information about global warming through a "partisan lens" decreased the correlation between cognitive sophistication and argument evaluation by decreasing polarization for those high in sophistication and increasing it for those low in sophistication. This result is hard to accommodate under a straightforward identity-protective cognition account. One possible explanation is that high and low skill reasoners may have different motivations and are thus differentially impacted by being asked to consider something through a political lens. Another possibility is that our manipulation did not increase political motivations in the way that was intended. For example, perhaps asking people to think about the information through a political lens surfaced their political biases, which people then suppressed. Naturally, if this is true then one is nonetheless left with the conclusion that political motivations are not as simple to induce as one might think, which implies that the impact of political motivations on science beliefs may be more complicated than the literature seems to indicate.

Interestingly, there was an association between polarization and cognitive sophistication when people evaluated climate change arguments under standard instructions (i.e., in the control condition of Studies 2 and 3), which supports the identity-protective cognition account.

However, it is unclear why this would essentially disappear when political motivations are made more salient. One possibility is that people who are higher in sophistication are more engaged with the task (i.e., more motivated) or have different or stronger prior beliefs (Bago et al., 2020; 
Tappin et al., 2020a, 2020b). A recent set of experiments found that experimentally increasing deliberation led to increased polarization in an evidence evaluation task that included pro- and anti-climate change arguments, but this primarily occurred because it increased the influence of prior beliefs in evidence evaluation (which can be a Bayesian process) (Bago et al., 2020). In any case, the ambiguity of the present results indicates that more research focused on manipulating underlying motivations is necessary.

Consistency with recent work on politically motivated reasoning. Our results are also broadly consistent with some recent work that has also begun to call politically motivated reasoning accounts into question (e.g., Druckman \& McGrath, 2019). For example, recent research has shown that partisan differences observed in survey research are often due to the desire for inter-party conflict and bias in the survey design rather than actual inter-party differences in beliefs (Bullock et al., 2015; Bullock \& Lenz, 2019; Roush \& Sood, 2020). Other work shows that people who are more analytic are better able to distinguish between true and false news regardless of consistency with political ideology (Pennycook \& Rand, 2019, 2021). Recent research on the increasingly polarized context of COVID-19 in the United States also shows that cognitive sophistication is associated with fewer misperceptions for Democrats and Republicans alike (despite Republicans being far more likely to hold misperceptions) (Pennycook et al., 2021).

Additionally, some research has found that providing more information does not cause a politically motivated "backfire effect" (Wood \& Porter, 2019) and, more directly, that communicating facts about the scientific consensus around climate change is effective for both conservatives and liberals (van der Linden et al., 2017). Indeed, in this latter study, van der Linden et al. (2017) found the pattern often used to support the identity-protective cognition 
account at pre-test; i.e., the educated were more polarized. However, once information about the climate consensus was provided, partisans (on both side of the aisle) increased their acceptance of climate change regardless of education levels. As with our own studies, this indicates that politically motivated reasoning may not play as strong a causal role in polarization around scientific issues as previously thought.

Importantly, these studies do not show that prior beliefs are not important in the context of people's evaluations about science. Rather, our evidence indicates that political ideology is not a particularly strong predictor for many science beliefs, which in turn implies that politically motivated reasoning does not offer as much of a challenge to science communication as previously thought. There are still contexts in which politics apparently has an influence on science beliefs - such as the case of global warming. However, even here, simple manipulations of political motivations (such as having people consider evidence through a political lens) do not apparently produce straightforward evidence of political polarization in evaluations. These findings combined indicate that the literature on science beliefs may need to be recalibrated.

The role of cognitive sophistication. There was not a single science belief where cognitive sophistication was consistently correlated in opposing directions for Democrats and Republicans across both samples in Study 1. This leads to our third major conclusion: thinking appears to rarely hurt. In fact, only in one instance was cognitive sophistication correlated negatively with the pro-science position: The claim that some transgender people re-transition genders. This item was intentionally worded to be obviously true (i.e., clearly some people retransition). However, it is also the case that only a very small proportion of people actually do retransition (Clarke \& Spilliadis, 2019) and so the item can be reasonably read as at least somewhat (or perhaps even largely) false in terms of its implication. Nonetheless, additional 
analyses excluding this item from the conservative pro-science composite yielded nearly identical results to those reported above (see Tables S48-S49). Thus, even in this one exceptional case, more cognitively sophisticated individuals may hold the more pro-scientific opinion. The present results therefore echo research demonstrating the important role of reflective thinking in belief formation and retention (Pennycook, Fugelsang, et al., 2015) and indicate that those higher in cognitive sophistication - that is, more able to think reflectively, openly, and skepticallygenerally hold more pro-science beliefs.

Knowledge about basic science is important and useful. Our final conclusion emerges from the finding that knowledge about basic science was broadly quite predictive of pro-science beliefs (and never predictive of anti-science beliefs). This finding has important policy implications. Put briefly: science can, and should, be taught. This may seem like an obvious recommendation, but recent research (Drummond \& Fischhoff, 2017b; Kahan et al., 2012) and articles in the popular press (Kahan, 2018; Nisbett, 2016) warn that more science knowledge may even have detrimental effects - indeed, this is also a key claim of the identity-protective cognition account (Drummond \& Fischhoff, 2017b; Kahan, 2013; Kahan et al., 2012).

Nonetheless, we find that basic knowledge of scientific facts (e.g., that electrons are smaller than atoms) was associated with pro-science beliefs even among politicized issues. Thus, with perhaps a few exceptions, focusing on teaching basic science is likely to yield increases in overall acceptance of science.

Our research extends past evidence suggesting that science education is a useful predictor of attitudes towards contentious science topics. A meta-analysis suggests that general science knowledge shows a small, but positive, correlation with attitudes towards climate and genetic sciences (Allum et al., 2011). Additionally, experimental research demonstrates effective 
strategies in this domain. For example, teaching people about the science behind genetically modified foods results in more positive attitudes (McPhetres et al., 2019), as does teaching people about the science behind climate change (Ranney \& Clark, 2016) — which is notable as this was the most consistently ideological topic in the present studies. Further, as noted, providing people with scientific information does not appear to cause a backfire effect, contrary to the prediction by the identity protective cognition account (van der Linden et al., 2017; Wood \& Porter, 2019). In contrast, beliefs about politics, morality, and religion are closely tied to one's identity and so are difficult to change. Thus, even on those few issues where political beliefs were more strongly correlated with attitudes, it may nonetheless be worthwhile to ensure that people understand the basic science.

\section{Concluding Comments}

Our results clarify the complex factors that help determine belief in particular scientific issues. These results suggest that political partisanship does not play as dominant a role as previously considered and that cognitive sophistication does not generally exacerbate identityprotective cognition, but is instead generally associated with pro-scientific beliefs. Instead of focusing on what divides people, we recommend that science communicators, educators, and policy-makers focus on improving science literacy to improve attitudes towards science and acceptance of new technologies. 


\section{Context of the Research}

This research originated out of desire to more comprehensively assess different perspectives across an increasingly fractured body of research on science beliefs and attitudes. The present authors viewed things from related but different perspectives (namely with a focus on science knowledge for Jonathon McPhetres and with a focus on analytic thinking for Gordon Pennycook and Bence Bago) and it was noteworthy in our discussions that there were still two other prominent perspectives about who holds anti-science beliefs (politically motivated reasoning and identity-protective cognition). For people who are focused on science communication and/or education, this lack of clarity in the literature could be very frustrating as different experts who focus on different components may offer extremely different perspectives on the same broad issue. At the very least, we would hope that research on the science of science beliefs would be less contentious than the beliefs themselves, and this paper is our attempt to synthesize and evaluate these competing perspectives.

\section{Author Note}

We gratefully acknowledge funding from the Ethics and Governance of Artificial Intelligence Initiative of the Miami Foundation, Reset (a project of Luminate), the John Templeton Foundation, and the Social Sciences and Humanities Research Council of Canada. This research was approved by the University of Regina Research Ethics Board. This research was disseminated as a working paper prior to publication (https://osf.io/ad9v7/) and was presented at the International Conference on Thinking in 2021.

\section{Data availability}

Data and materials: https://osf.io/h4ej5/?view_only=a96965ef6e8342eab99fe446d3fba198

Preregistration for Study 1, Sample 1:

https://osf.io/s9n24/?view_only=06204d2bc9ea496db7d80fa15706aa2b

Preregistration for Study 1, Sample 2:

https://osf.io/5tr2f/?view_only=ea980de25a68437c928211b2b1d01a6d

Preregistration for pilot: https://osf.io/wsz7e/?view_only=d13810b9e9ca40a9adbc269a019e97f8

Preregistrations for Studies 2 and 3: https://osf.io/h4ej5/ 


\section{References}

Abramowitz, A. I. (2010). The Disappearing Center. In The Disappearing Center. Yale University Press. https://doi.org/10.12987/9780300162882/HTML

Allum, N., Sturgis, P., Tabourazi, D., Brunton-smith, I., Allum, N., Sturgis, P., Tabourazi, D., Science, I. B., Allum, N., Sturgis, P., Tabourazi, D., \& Brunton-smith, I. (2011). Science knowledge and attitudes across cultures : a To cite this version: Science knowledge and attitudes across cultures : a meta-analysis. 17, 35-54.

https://doi.org/11.1077/0963662506070159

Bago, B., Rand, D. G., \& Pennycook, G. (2020). Reasoning about climate change. PsyArXiv, 122. https://doi.org/10.31234/OSF.IO/VCPKB

Barr, D. J., Levy, R., Scheepers, C., \& Tily, H. J. (2013). Random effects structure for confirmatory hypothesis testing: Keep it maximal. Journal of Memory and Language, 68(3), 255-278. https://doi.org/10.1016/j.jml.2012.11.001

Bohr, J. (2014). Public views on the dangers and importance of climate change: predicting climate change beliefs in the United States through income moderated by party identification. Climatic Change, 126(1-2), 217-227. https://doi.org/10.1007/s10584-014$1198-9$

Bolsen, T., Druckman, J. N., \& Cook, F. L. (2014). The Influence of Partisan Motivated Reasoning on Public Opinion. Political Behavior, 36(2), 235-262. https://doi.org/10.1007/S11109-013-9238-0/FIGURES/3

Bullock, J. G., Gerber, A. S., Hill, S. J., \& Huber, G. (2015). Partisan bias in factual beliefs about politics. Quarterly Journal of Political Science, 10(4), 519-578.

https://doi.org/10.1561/100.00014074 
Bullock, J. G., \& Lenz, G. (2019). Partisan Bias in Surveys. Annual Review of Political Science, 22(1), 325-342. https://doi.org/10.1146/annurev-polisci-051117-050904

Clarke, A. C., \& Spilliadis, A. (2019). 'Taking the lid off the box': The value of extended clinical assessment for adolescents presenting with gender identity difficulties. Clinical Child Psychology and Psychiatry, 24(2), 3380352. https://doi.org/https://doi.org/10.1177/1359104518825288

Druckman, J. N., \& McGrath, M. C. (2019). The evidence for motivated reasoning in climate change preference formation. Nature Climate Change, 9(2), 111-119. https://doi.org/10.1038/s41558-018-0360-1

Drummond, C., \& Fischhoff, B. (2017a). Individuals with greater science literacy and education have more polarized beliefs on controversial science topics. Proceedings of the National Academy of Sciences, 114, 9587-9592. https://doi.org/10.1073/pnas.1704882114

Drummond, C., \& Fischhoff, B. (2017b). Development and Validation of the Scientific Reasoning Scale. Journal of Behavioral Decision Making, 30(1), 26-38. https://doi.org/10.1002/bdm.1906

Ehret, P. J., Sparks, A. C., \& Sherman, D. K. (2017). Support for environmental protection: an integration of ideological-consistency and information-deficit models. Environmental Politics, 26(2), 253-277. https://doi.org/10.1080/09644016.2016.1256960

Erisen, C., Lodge, M., \& Taber, C. S. (2014). Affective contagion in effortful political thinking. Political Psychology, 35(2), 187-206. https://doi.org/10.1111/j.1467-9221.2012.00937.x

Frederick, S. (2005). Cognitive Reflection and Decision Making. Journal of Economic Perspectives, 19(4), 25-42. https://doi.org/10.1257/089533005775196732

Funder, D. C., \& Ozer, D. J. (2019). Evaluating Effect Size in Psychological Research: Sense 
and Nonsense. Advances in Methods and Practices in Psychological Science, 251524591984720. https://doi.org/10.1177/2515245919847202

Gauchat, G. (2012). Politicization of Science in the Public Sphere: A Study of Public Trust in the United States, 1974 to 2010. American Sociological Review, 77(2), 167-187. https://doi.org/10.1177/0003122412438225

Gervais, W. M. (2015). Override the controversy: Analytic thinking predicts endorsement of evolution. Cognition, 142, 312-321. https://doi.org/10.1016/j.cognition.2015.05.011

Gignac, G. E., \& Szodorai, E. T. (2016). Effect size guidelines for individual differences researchers. Personality and Individual Differences, 102, 74-78. https://doi.org/10.1016/j.paid.2016.06.069

Guilbeault, D., Becker, J., \& Centola, D. (2018). Social learning and partisan bias in the interpretation of climate trends. Proceedings of the National Academy of Sciences of the United States of America, 115(39), 9714-9719. https://doi.org/10.1073/pnas.1722664115

Hill, J. P. (2014). Rejecting Evolution: The Role of Religion, Education, and Social Networks. Journal for the Scientific Study of Religion, 53(3), 575-594. https://doi.org/10.1111/JSSR.12127

Iyengar, S., \& Massey, D. S. (2019). Scientific communication in a post-truth society. Proceedings of the National Academy of Sciences of the United States of America, 116(16), 7656-7661. https://doi.org/10.1073/pnas.1805868115

Joslyn, M. R., \& Sylvester, S. M. (2019). The Determinants and Consequences of Accurate Beliefs About Childhood Vaccinations. American Politics Research, 47(3), 628-649. https://doi.org/10.1177/1532673X17745342

Kahan, D. M. (2013). Ideology, motivated reasoning, and cognitive reflection. Judgment and 
Decision Making, 8(4), 407-424. https://doi.org/10.2139/ssrn.2182588

Kahan, D. M. (2015). Climate science communication and the measurement problem. Advances Political Psychology, 36, 1-43. https://doi.org/10.1111/pops.12244

Kahan, D. M. (2018). Why Smart People Are Vulnerable to Putting Tribe Before Truth. Scientific American.

Kahan, D. M., Landrum, A., Carpenter, K., Helft, L., \& Hall Jamieson, K. (2017). Science Curiosity and Political Information Processing. Political Psychology, 38, 179-199. https://doi.org/10.1111/pops.12396

Kahan, D. M., Peters, E., Wittlin, M., Slovic, P., Ouellette, L. L., Braman, D., \& Mandel, G. (2012). The polarizing impact of science literacy and numeracy on perceived climate change risks. Nature Climate Change, 2(10), 732-735. https://doi.org/10.1038/nclimate1547

Kunda, Z. (1990). The case for motivated reasoning. Psychological Bulletin, 108(3), 480-498. https://doi.org/10.1037/0033-2909.108.3.480

Lewandowsky, S., Oberauer, K., \& Gignac, G. E. (2013). NASA Faked the Moon LandingTherefore, (Climate) Science Is a Hoax An Anatomy of the Motivated Rejection of Science. Psychological Science, 24(5), 622-633. https://doi.org/10.1177/0956797612457686

Lodge, M., \& Taber, C. (2005). The Automaticity of Affect for Political Candidates, Parties and Issues. Political Psychology, 26(3), 455-482.

Lombrozo, T., Thanukos, A., \& Weisberg, M. (2008). The Importance of Understanding the Nature of Science for Accepting Evolution. Evolution: Education and Outreach, 1(3), 290298. https://doi.org/10.1007/s12052-008-0061-8

Mccright, A. M., \& Dunlap, R. E. (2011). The Politicization Of Climate Change And 
Polarization In The American Public's Views Of Global Warming, 2001-2010. Sociological Quarterly, 52(2), 155-194. https://doi.org/10.1111/j.1533-8525.2011.01198.x

McCright, A. M., Marquart-Pyatt, S. T., Shwom, R. L., Brechin, S. R., \& Allen, S. (2016). Ideology, capitalism, and climate: Explaining public views about climate change in the United States. Energy Research and Social Science, 21, 180-189. https://doi.org/10.1016/j.erss.2016.08.003

McPhetres, J., Rutjens, B. T., Weinstein, N., \& Brisson, J. A. (2019). Modifying attitudes about modified foods: Increased knowledge leads to more positive attitudes. Journal of Environmental Psychology, 64(May), 21-29. https://doi.org/10.1016/j.jenvp.2019.04.012

Nisbett, M. (2016). The Science Literacy Paradox. Skeptical Inquirer, 40(5).

O’Brien, T. C., Palmer, R., \& Albarracin, D. (2021). Misplaced trust: When trust in science fosters belief in pseudoscience and the benefits of critical evaluation. Journal of Experimental Social Psychology, 96, 104184. https://doi.org/10.1016/J.JESP.2021.104184

Pennycook, G., Cheyne, J. A., Barr, N., Koehler, D. J., \& Fugelsang, J. A. (2015). On the reception and detection of pseudo-profound bullshit. Judgment and Decision Making, 10(6), $549-563$.

Pennycook, G., Cheyne, J. A., Koehler, D. J., \& Fugelsang, J. A. (2020). On the belief that beliefs should change according to evidence: Implications for conspiratorial, moral, paranormal, political, religious, and science beliefs. Judgment and Decision Making, 15, 476-498. https://doi.org/10.31234/OSF.IO/A7K96

Pennycook, G., Fugelsang, J. A., \& Koehler, D. J. (2015). Everyday Consequences of Analytic Thinking. Current Directions in Psychological Science, 24(6), 425-432. https://doi.org/10.1177/0963721415604610 
Pennycook, G., McPhetres, J., Bago, B., \& Rand, D. G. (2021). Beliefs About COVID-19 in Canada, the United Kingdom, and the United States: A Novel Test of Political Polarization and Motivated Reasoning. Personality and Social Psychology Bulletin, 014616722110236. https://doi.org/10.1177/01461672211023652

Pennycook, G., \& Rand, D. G. (2019). Lazy, not biased: Susceptibility to partisan fake news is better explained by lack of reasoning than by motivated reasoning. Cognition, 188, 39-50. https://doi.org/10.1016/j.cognition.2018.06.011

Pennycook, G., \& Rand, D. G. (2021). The Psychology of Fake News. Trends in Cognitive Sciences, 1-29. https://doi.org/10.1016/j.tics.2021.02.007

Prior, M. (2013). Media and Political Polarization. Annual Review of Political Science, 16, 101127. https://doi.org/10.1146/ANNUREV-POLISCI-100711-135242

Ranney, M. A., \& Clark, D. (2016). Climate Change Conceptual Change: Scientific Information Can Transform Attitudes. Topics in Cognitive Science, 8(1), 49-75. https://doi.org/10.1111/tops.12187

Roush, C. E., \& Sood, G. (2020). A Gap in Our Understanding? Reconsidering the Evidence for Partisan Knowledge Gaps. Unpublished Manuscript.

Rutjens, B. T., Heine, S. J., Sutton, R. M., \& van Harreveld, F. (2017). Attitudes Towards Science. 57, 1-49. https://doi.org/10.1016/bs.aesp.2017.08.001

Rutjens, B. T., Sutton, R. M., \& van der Lee, R. (2018). Not All Skepticism Is Equal: Exploring the Ideological Antecedents of Science Acceptance and Rejection. Personality and Social Psychology Bulletin, 44(3), 384-405. https://doi.org/10.1177/0146167217741314

Scheufele, D. A. (2013). Communicating science in social settings. Proceedings of the National Academy of Sciences of the United States of America, 110(SUPPL. 3), 14040-14047. 
https://doi.org/10.1073/pnas.1213275110

Scheufele, D. A. (2014). Science communication as political communication. Proceedings of the National Academy of Sciences of the United States of America, 111(Supplement 4), 1358513592. https://doi.org/10.1073/pnas.1317516111

Taber, C. S., Cann, D., \& Kucsova, S. (2009). The motivated processing of political arguments. Political Behavior, 31(2), 137-155. https://doi.org/10.1007/s11109-008-9075-8

Taber, C. S., \& Lodge, M. (2006). Motivated Skepticism in the Evaluation of Political Beliefs. American Journal of Political Science, 50(3), 755-769. https://doi.org/10.1111/J.15405907.2006.00214.X

Taber, C. S., \& Lodge, M. (2012). Motivated skepticism in the evaluation of political beliefs (2006). Critical Review, 24(2), 157-184. https://doi.org/10.1080/08913811.2012.711019

Tappin, B. M., Pennycook, G., \& Rand, D. G. (2020a). Bayesian or biased? Analytic thinking and political belief updating. Cognition. https://psyarxiv.com/a6euj/

Tappin, B. M., Pennycook, G., \& Rand, D. G. (2020b). Rethinking the link between cognitive sophistication and politically motivated reasoning. Journal of Experimental Psychology: General. https://doi.org/10.31234/OSF.IO/YUZFJ

van der Linden, S., Leiserowitz, A., \& Maibach, E. (2017). Scientific agreement can neutralize politicization of facts. Nature Human Behaviour. https://doi.org/10.1038/s41562-017-02592

Vitriol, J. A., Lavine, H. G., \& Borgida, E. (2020). Meta-cognition and resistance to political persuasion: evidence from a three-wave panel study. Social Influence, 15(1), 17-33. https://doi.org/10.1080/15534510.2020.1760129/SUPPL_FILE/PSIF_A_1760129_SM9105 .DOCX 
Weisberg, D. S., Landrum, A. R., Hamilton, J., \& Weisberg, M. (2021). Knowledge about the nature of science increases public acceptance of science regardless of identity factors. Public Understanding of Science, 30(2), 120-138.

https://doi.org/10.1177/0963662520977700

Weisberg, D. S., Landrum, A. R., Metz, S. E., \& Weisberg, M. (2018). No Missing Link: Knowledge Predicts Acceptance of Evolution in the United States. BioScience, 68(3), 212222. https://doi.org/10.1093/BIOSCI/BIX161

Wood, T., \& Porter, E. (2019). The elusive backfire effect: Mass attitudes' steadfast factual adherence. Political Behavior, 41(1), 135-163.

Zaller, J. R. (1992). The Nature and Origins of Mass Opinion. In The Nature and Origins of Mass Opinion. Cambridge University Press. https://doi.org/10.1017/cbo9780511818691

Funding: This research was supported by the Social Sciences and Humanities Research Council of Canada, the John Templeton Foundation, and the Miami Foundation. BB was supported by the ANR grant ANR-17-EURE-0010 (Investissements d'Avenir program), and ANR Labex IAST.

Author Contributions: JM and GP designed the study and wrote the manuscript; JM conducted data analysis.

Competing Interests: All authors declare no competing interests.

Data Availability: Our analysis plans, inclusion criteria, and study design were preregistered prior to data collection; all data and materials are available on the Open Science Framework (https://osf.io/h4ej5/?view_only=a96965ef6e8342eab99fe446d3fba198). The preregistration for Sample 1 is available at: https://osf.io/s9n24/?view_only=06204d2bc9ea496db7d80fa15706aa2b. The preregistration for Sample 2 is available at: https://osf.io/5tr2f/?view_only=ea980de25a68437c928211b2b1d01a6d. The preregistration for the pilot sample is available at: https://osf.io/wsz7e/?view_only=d13810b9e9ca40a9adbc269a019e97f8. The preregistrations for Studies 2 and 3 are available at: https://osf.io/h4ej5/?view_only=a96965ef6e8342eab99fe446d3fba198. 